\title{
Properties of Matrix Variate Confluent Hypergeometric Function Distribution
}

\author{
Arjun K. Gupta, ${ }^{1}$ Daya K. Nagar, ${ }^{2}$ and Luz Estela Sánchez ${ }^{2}$ \\ ${ }^{1}$ Department of Mathematics and Statistics, Bowling Green State University, Bowling Green, OH 43403-0221, USA \\ ${ }^{2}$ Instituto de Matemáticas, Universidad de Antioquia, Calle 67, No. 53-108, Medellín, Colombia
}

Correspondence should be addressed to Daya K. Nagar; dayaknagar@yahoo.com

Received 3 September 2015; Accepted 15 December 2015

Academic Editor: Z. D. Bai

Copyright (C) 2016 Arjun K. Gupta et al. This is an open access article distributed under the Creative Commons Attribution License, which permits unrestricted use, distribution, and reproduction in any medium, provided the original work is properly cited.

We study matrix variate confluent hypergeometric function kind 1 distribution which is a generalization of the matrix variate gamma distribution. We give several properties of this distribution. We also derive density functions of $X_{2}^{-1 / 2} X_{1} X_{2}^{-1 / 2},\left(X_{1}+X_{2}\right)^{-1 / 2} X_{1}\left(X_{1}+\right.$ $\left.X_{2}\right)^{-1 / 2}$, and $X_{1}+X_{2}$, where $m \times m$ independent random matrices $X_{1}$ and $X_{2}$ follow confluent hypergeometric function kind 1 and gamma distributions, respectively.

\section{Introduction}

The matrix variate gamma distribution has many applications in multivariate statistical analysis. The Wishart distribution, which is the distribution of the sample variance covariance matrix when sampling from a multivariate normal distribution, is a special case of the matrix variate gamma distribution.

The purpose of this paper is to give a generalization of the matrix variate gamma distribution and study its properties.

We begin with a brief review of some definitions and notations. We adhere to standard notations (cf. Gupta and Nagar [1]). Let $A=\left(a_{i j}\right)$ be an $m \times m$ matrix. Then, $A^{\prime}$ denotes the transpose of $A ; \operatorname{tr}(A)=a_{11}+\cdots+a_{m m}$; $\operatorname{etr}(A)=$ $\exp (\operatorname{tr}(A)) ; \operatorname{det}(A)=\operatorname{determinant}$ of $A$; norm of $A=\|A\|=$ maximum of absolute values of eigenvalues of the matrix $A$; $A>0$ means that $A$ is symmetric positive definite; and $A^{1 / 2}$ denotes the unique symmetric positive definite square root of $A>0$. The multivariate gamma function $\Gamma_{m}(a)$ is defined by

$$
\begin{aligned}
\Gamma_{m}(a) & =\int_{X>0} \operatorname{etr}(-X) \operatorname{det}(X)^{a-(m+1) / 2} \mathrm{~d} X \\
& =\pi^{m(m-1) / 4} \prod_{i=1}^{m} \Gamma\left(a-\frac{i-1}{2}\right), \quad \operatorname{Re}(a)>\frac{m-1}{2} .
\end{aligned}
$$

The $m \times m$ symmetric positive definite random matrix $X$ is said to have a matrix variate gamma distribution, denoted by
$X \sim \mathrm{Ga}(m, v, \theta, \Omega)$, if its probability density function (p.d.f.) is given by

$$
\frac{\operatorname{det}(X)^{\nu-(m+1) / 2} \operatorname{etr}\left(-\Omega^{-1} X / \theta\right)}{\Gamma_{m}(\nu) \theta^{m v} \operatorname{det}(\Omega)^{\nu}}, \quad X>0,
$$

where $\Omega$ is a symmetric positive definite matrix of order $m, \theta>0$, and $v>(m-1) / 2$. For $\Omega=I_{m}$, the above density reduces to a standard matrix variate gamma density and in this case we write $X \sim \mathrm{Ga}(m, v, \theta)$. Further, if $X_{1} \sim$ $\mathrm{Ga}\left(m, v_{1}, \theta\right)$ and $X_{2} \sim \mathrm{Ga}\left(m, v_{2}, \theta\right)$ are independent gamma matrices, then the random matrix $\left(X_{1}+X_{2}\right)^{-1 / 2} X_{1}\left(X_{1}+\right.$ $\left.X_{2}\right)^{-1 / 2}$ follows a matrix variate beta type 1 distribution with parameters $\nu_{1}$ and $\nu_{2}$.

By replacing $\operatorname{etr}\left(-\Omega^{-1} X / \theta\right)$ by the confluent hypergeometric function of matrix argument ${ }_{1} F_{1}\left(\alpha ; \beta ;-\Omega^{-1} X / \theta\right)$, a generalization of the matrix variate gamma distribution can be defined by the p.d.f.:

$$
C(\nu, \alpha, \beta, \theta, \Omega) \operatorname{det}(X)^{\nu-(m+1) / 2}{ }_{1} F_{1}\left(\alpha ; \beta ;-\frac{1}{\theta} \Omega^{-1} X\right),
$$

where $X>0$ and $C(\nu, \alpha, \beta, \theta, \Omega)$ is the normalizing constant. In Section 2, it has been shown that, for $\beta-v>(m-1) / 2$, 
$\alpha-\nu>(m-1) / 2, \nu>(m-1) / 2, \theta>0$, and $\Omega>0$, the normalizing constant can be evaluated as

$$
C(\nu, \alpha, \beta, \theta, \Omega)=\frac{\Gamma_{m}(\alpha) \Gamma_{m}(\beta-\nu) \operatorname{det}(\theta \Omega)^{-v}}{\Gamma_{m}(\nu) \Gamma_{m}(\beta) \Gamma_{m}(\alpha-\nu)} .
$$

Therefore, the p.d.f. in (3) can be written explicitly as

$$
\begin{gathered}
\frac{\Gamma_{m}(\alpha) \Gamma_{m}(\beta-\nu) \operatorname{det}(\theta \Omega)^{-\nu}}{\Gamma_{m}(\nu) \Gamma_{m}(\beta) \Gamma_{m}(\alpha-\nu)} \operatorname{det}(X)^{\nu-(m+1) / 2} \\
\cdot{ }_{1} F_{1}\left(\alpha ; \beta ;-\frac{1}{\theta} \Omega^{-1} X\right), \quad X>0,
\end{gathered}
$$

where $\beta-v>(m-1) / 2, \alpha-v>(m-1) / 2, v>(m-1) / 2, \theta>0$, $\Omega>0$, and ${ }_{1} F_{1}$ is the confluent hypergeometric function of the first kind of matrix argument (Gupta and Nagar [1]). Since the density given above involves the confluent hypergeometric function, we will call the corresponding distribution a confluent hypergeometric function distribution. We will write $X \sim \mathrm{CH}_{m}(\nu, \alpha, \beta, \theta, \Omega$, kind 1) to say that the random matrix $X$ has a confluent hypergeometric function distribution defined by the density (5). It has been shown by van der Merwe and Roux [2] that the above density can be obtained as a limiting case of a density involving the Gauss hypergeometric function of matrix argument. For $\alpha=\beta$, the density (5) reduces to a matrix variate gamma density and for $\Omega=I_{m}$ it slides to

$$
\begin{gathered}
\frac{\Gamma_{m}(\alpha) \Gamma_{m}(\beta-\nu)}{\theta^{m \nu} \Gamma_{m}(\nu) \Gamma_{m}(\beta) \Gamma_{m}(\alpha-\nu)} \operatorname{det}(X)^{\nu-(m+1) / 2} \\
\cdot{ }_{1} F_{1}\left(\alpha ; \beta ;-\frac{1}{\theta} X\right), \quad X>0,
\end{gathered}
$$

where $\beta-v>(m-1) / 2, \alpha-v>(m-1) / 2, v>(m-1) / 2$, and $\theta>0$. In this case we will write $X \sim \mathrm{CH}_{m}(\nu, \alpha, \beta, \theta$, kind 1). The matrix variate confluent hypergeometric function kind 1 distribution occurs as the distribution of the matrix ratio of independent gamma and beta matrices. For $m=1,(6)$ reduces to a univariate confluent hypergeometric function kind 1 density given by (Orozco-Castañeda et al. [3])

$$
\frac{\Gamma(\alpha) \Gamma(\beta-\nu)}{\theta^{\nu} \Gamma(\nu) \Gamma(\beta) \Gamma(\alpha-\nu)} x_{1}^{\nu-1} F_{1}\left(\alpha ; \beta ;-\frac{x}{\theta}\right), \quad x>0,
$$

where $\beta-v>0, \alpha-\nu>0, \nu>0, \theta>0$, and ${ }_{1} F_{1}$ is the confluent hypergeometric function of the first kind (Luke [4]). The random variable $x$ having the above density will be designated by $x \sim \mathrm{CH}(\nu, \alpha, \beta, \theta$, kind 1$)$. Since the matrix variate confluent hypergeometric function kind 1 distribution is a generalization of the matrix variate gamma distribution, it is reasonable to say that the matrix variate confluent hypergeometric function kind 1 distribution can be used as an alternative to the gamma distribution quite effectively.

Although ample information about matrix variate gamma distribution is available, little appears to have been done in the literature to study the matrix variate confluent hypergeometric function kind 1 distribution.
In this paper, we study several properties including stochastic representations of the matrix variate confluent hypergeometric function kind 1 distribution. We also derive the density function of the matrix quotient of two independent random matrices having confluent hypergeometric function kind 1 and gamma distributions. Further, densities of several other matrix quotients and matrix products involving confluent hypergeometric function kind 1, beta type 1 , beta type 2 , and gamma matrices are derived.

\section{Some Definitions and Preliminary Results}

In this section we give some definitions and preliminary results which are used in subsequent sections.

A more general integral representation of the multivariate gamma function can be obtained as

$$
\Gamma_{m}(a)=\operatorname{det}(Y)^{a} \int_{R>0} \operatorname{etr}(-Y R) \operatorname{det}(R)^{a-(m+1) / 2} \mathrm{~d} R
$$

where $\operatorname{Re}(a)>(m-1) / 2$ and $\operatorname{Re}(Y)>(m-1) / 2$. The above result can be established for real $Y>0$ by substituting $X=$ $Y^{1 / 2} R Y^{1 / 2}$ with the Jacobian $J(X \rightarrow R)=\operatorname{det}(Y)^{(m+1) / 2}$ in (1) and it follows for complex $Y$ by analytic continuation.

The multivariate generalization of the beta function is given by

$$
\begin{aligned}
B_{m} & (a, b) \\
& =\int_{0}^{I_{m}} \operatorname{det}(R)^{a-(m+1) / 2} \operatorname{det}\left(I_{m}-R\right)^{b-(m+1) / 2} \mathrm{~d} R \\
& =\frac{\Gamma_{m}(a) \Gamma_{m}(b)}{\Gamma_{m}(a+b)}=B_{m}(b, a),
\end{aligned}
$$

where $\operatorname{Re}(a)>(m-1) / 2$ and $\operatorname{Re}(b)>(m-1) / 2$.

The generalized hypergeometric function of one matrix, defined in Constantine [5], is given by

$$
\begin{aligned}
{ }_{p} F_{q} & \left(a_{1}, \ldots, a_{p} ; b_{1}, \ldots, b_{q} ; X\right) \\
& =\sum_{k=0}^{\infty} \sum_{\kappa \vdash k} \frac{\left(a_{1}\right)_{\kappa} \cdots\left(a_{p}\right)_{\kappa}}{\left(b_{1}\right)_{\kappa} \cdots\left(b_{q}\right)_{\kappa}} \frac{C_{\kappa}(X)}{k !}
\end{aligned}
$$

where $a_{i}, i=1, \ldots, p, b_{j}, j=1, \ldots, q$ are arbitrary complex numbers, $X$ is an $m \times m$ complex symmetric matrix, $C_{\kappa}(X)$ is the zonal polynomial of $m \times m$ complex symmetric matrix $X$ corresponding to the ordered partition $\kappa=\left(k_{1}, \ldots, k_{m}\right)$, $k_{1} \geq \cdots \geq k_{m} \geq 0, k_{1}+\cdots+k_{m}=k$, and $\sum_{\kappa \vdash k}$ denotes the summation over all partitions $\kappa$. The generalized hypergeometric coefficient $(a)_{\kappa}$ used above is defined by

$$
(a)_{\kappa}=\prod_{i=1}^{m}\left(a-\frac{i-1}{2}\right)_{k_{i}}
$$


where $(a)_{r}=a(a+1) \cdots(a+r-1), r=1,2, \ldots$, with $(a)_{0}=1$. Conditions for convergence of the series in (10) are available in the literature. From (10), it follows that

$$
\begin{aligned}
{ }_{0} F_{0}(X) & =\sum_{k=0}^{\infty} \sum_{\kappa \vdash k} \frac{C_{\kappa}(X)}{k !}=\operatorname{etr}(X), \\
{ }_{1} F_{0}(a ; X) & =\sum_{k=0}^{\infty} \sum_{\kappa \vdash k} \frac{(a)_{\mathcal{K}} C_{\kappa}(X)}{k !} \\
& =\operatorname{det}\left(I_{m}-X\right)^{-a}, \quad\|X\|<1, \\
{ }_{1} F_{1}(a ; c ; X) & =\sum_{k=0}^{\infty} \sum_{\kappa \vdash k} \frac{(a)_{\kappa} C_{\kappa}(X)}{(c)_{\kappa} k !}, \\
{ }_{2} F_{1}(a, b ; c ; X) & =\sum_{k=0}^{\infty} \sum_{\kappa \vdash k} \frac{(a)_{\kappa}(b)_{\kappa}}{(c)_{\kappa}} \frac{C_{\kappa}(X)}{k !}, \quad\|X\|<1 .
\end{aligned}
$$

By taking $a_{p}=b_{q}=c$ in (10), it can be observed that

$$
\begin{aligned}
{ }_{p} F_{q} & \left(a_{1}, \ldots, a_{p-1}, c ; b_{1}, \ldots, b_{q-1}, c ; X\right) \\
& ={ }_{p-1} F_{q-1}\left(a_{1}, \ldots, a_{p-1} ; b_{1}, \ldots, b_{q-1} ; X\right) .
\end{aligned}
$$

Substituting $p=2, q=1$ in (15) and using (12), the Gauss hypergeometric function ${ }_{2} F_{1}(a, c ; c ; X)$ is reduced as

$$
{ }_{2} F_{1}(a, c ; c ; X)={ }_{1} F_{0}(a ; X)=\operatorname{det}\left(I_{m}-X\right)^{-a},
$$

$\|X\|<1$.

The integral representations of the confluent hypergeometric function ${ }_{1} F_{1}$ and the Gauss hypergeometric function ${ }_{2} F_{1}$ are given by

$$
\begin{aligned}
& { }_{1} F_{1}(a ; c ; X)=\frac{1}{B_{m}(a, c-a)} \int_{0}^{I_{m}} \operatorname{det}(R)^{a-(m+1) / 2} \\
& \cdot \operatorname{det}\left(I_{m}-R\right)^{c-a-(m+1) / 2} \operatorname{etr}(X R) \mathrm{d} R, \\
& { }_{2} F_{1}(a, b ; c ; X)=\frac{1}{B_{m}(a, c-a)} \\
& \cdot \int_{0}^{I_{m}} \frac{\operatorname{det}(R)^{a-(m+1) / 2} \operatorname{det}\left(I_{m}-R\right)^{c-a-(m+1) / 2}}{\operatorname{det}\left(I_{m}-X R\right)^{b}} \mathrm{~d} R,
\end{aligned}
$$

where $\operatorname{Re}(a)>(m-1) / 2$ and $\operatorname{Re}(c-a)>(m-1) / 2$.

Further generalizations of (8) and (9) in terms of zonal polynomials, due to Constantine [5], are given as

$$
\begin{aligned}
& \int_{R>0} \operatorname{etr}(-Y R) \operatorname{det}(R)^{a-(m+1) / 2} C_{\kappa}(X R) \mathrm{d} R \\
& =\Gamma_{m}(a)(a)_{\kappa} \operatorname{det}(Y)^{-a} C_{\kappa}\left(Y^{-1} X\right), \\
& \int_{0}^{I_{m}} \operatorname{det}(R)^{a-(m+1) / 2} \operatorname{det}\left(I_{m}-R\right)^{b-(m+1) / 2} C_{\kappa}(X R) \mathrm{d} R \\
& =\frac{B_{m}(a, b)(a)_{\kappa}}{(a+b)_{\kappa}} C_{\kappa}(X),
\end{aligned}
$$

respectively.
For $\operatorname{Re}(\alpha)>(m-1) / 2$ and $\operatorname{Re}(\beta)>(m-1) / 2$, we have

$$
\begin{aligned}
& \int_{R>0} \operatorname{det}(R)^{\alpha-(m+1) / 2} \operatorname{etr}(-Y R){ }_{p} F_{q}\left(a_{1}, \ldots, a_{p} ; b_{1}, \ldots, b_{q} ;\right. \\
& X R) \mathrm{d} R=\Gamma_{m}(\alpha) \operatorname{det}(Y)^{-a}{ }_{p+1} F_{q}\left(\alpha, a_{1}, \ldots, a_{p} ;\right. \\
& \left.b_{1}, \ldots, b_{q} ; X Y^{-1}\right), \\
& \int_{0}^{I_{m}} \operatorname{det}(R)^{\alpha-(m+1) / 2} \operatorname{det}\left(I_{m}\right. \\
& \quad-R)^{\beta-(m+1) / 2}{ }_{p} F_{q}\left(a_{1}, \ldots, a_{p} ; b_{1}, \ldots, b_{q} ; X R\right) \mathrm{d} R \\
& =B_{m}(\alpha, \beta)_{p+1} F_{q+1}\left(\alpha, a_{1}, \ldots, a_{p} ; \alpha+\beta, b_{1}, \ldots, b_{q} ; X\right) .
\end{aligned}
$$

We can establish (21) and (22) by expanding ${ }_{p} F_{q}$ in series form by using (10) and integrating term by term by applying (19) and (20) and finally summing the resulting series.

Note that the series expansions for ${ }_{1} F_{1}$ and ${ }_{2} F_{1}$ given in (13) and (14) can be obtained by expanding $\operatorname{etr}(X R)$ and $\operatorname{det}\left(I_{m}-X R\right)^{-b},\|X R\|<1$, in (17) and (18) and integrating $R$ using (20). Substituting $X=I_{m}$ in (18) and integrating, we obtain

$$
{ }_{2} F_{1}\left(a, b ; c ; I_{m}\right)=\frac{\Gamma_{m}(c) \Gamma_{m}(c-a-b)}{\Gamma_{m}(c-a) \Gamma_{m}(c-b)},
$$

where $\operatorname{Re}(c-a-b)>(m-1) / 2, \operatorname{Re}(c-a)>(m-1) / 2, \operatorname{Re}(c-$ $b)>(m-1) / 2$, and $\operatorname{Re}(c)>(m-1) / 2$. The hypergeometric function ${ }_{1} F_{1}(a ; c ; X)$ satisfies Kummer's relation

$$
{ }_{1} F_{1}(a ; c ;-X)=\operatorname{etr}(-X){ }_{1} F_{1}(c-a ; c ; X) .
$$

For properties and further results on these functions the reader is referred to Constantine [5], James [6], Muirhead [7], and Gupta and Nagar [1]. The numerical computation of a hypergeometric function of matrix arguments is very difficult. However, some numerical methods are proposed in recent years; see, Hashiguchi et al. [8] and Koev and Edelman [9].

The generalized hypergeometric function with $m \times m$ complex symmetric matrices $X$ and $Y$ is defined by

$$
\begin{aligned}
{ }_{p} F_{q}^{(m)} & \left(a_{1}, \ldots, a_{p} ; b_{1}, \ldots, b_{q} ; X, Y\right) \\
= & \sum_{k=0}^{\infty} \sum_{\kappa \vdash k} \frac{\left(a_{1}\right)_{\kappa} \cdots\left(a_{p}\right)_{\kappa}}{\left(b_{1}\right)_{\kappa} \cdots\left(b_{q}\right)_{\kappa}} \frac{C_{\kappa}(X) C_{\kappa}(Y)}{C_{\kappa}\left(I_{m}\right) k !} .
\end{aligned}
$$

It is clear from the above definition that the order of $X$ and $Y$ is unimportant; that is,

$$
\begin{aligned}
{ }_{p} F_{q}^{(m)}\left(a_{1}, \ldots, a_{p} ; b_{1}, \ldots, b_{q} ; X, Y\right) \\
\quad={ }_{p} F_{q}^{(m)}\left(a_{1}, \ldots, a_{p} ; b_{1}, \ldots, b_{q} ; Y, X\right) .
\end{aligned}
$$

Also, if one of the argument matrices is the identity, this function reduces to the one argument function. Further, the two-matrix argument function ${ }_{p} F_{q}^{(m)}$ can be obtained from the one-matrix function ${ }_{p} F_{q}$ by averaging over the orthogonal 
group $O(m)$ using a result given in James [6, Equation 23]; namely,

$$
\int_{O(m)} C_{\kappa}\left(X H Y H^{\prime}\right)(\mathrm{d} H)=\frac{C_{\kappa}(X) C_{\kappa}(Y)}{C_{\kappa}\left(I_{m}\right)},
$$

where $(\mathrm{d} H)$ denotes the normalized invariant measure on $O(m)$. That is,

$$
\begin{aligned}
& { }_{p} F_{q}^{(m)}\left(a_{1}, \ldots, a_{p} ; b_{1}, \ldots, b_{q} ; X, Y\right) \\
& =\int_{O(m)}{ }_{p} F_{q}^{(m)}\left(a_{1}, \ldots, a_{p} ; b_{1}, \ldots, b_{q} ; X H Y H^{\prime}\right)(\mathrm{d} H),
\end{aligned}
$$

given in James [6, Equation 30].

Finally, we define the inverted matrix variate gamma, matrix variate beta type 1 , and matrix variate beta type 2 distributions. These definitions can be found in Gupta and Nagar [1] and Iranmanesh et al. [10].

Definition 1 . An $m \times m$ random symmetric positive definite matrix $X$ is said to have an inverted matrix variate gamma distribution with parameters $\mu, \theta$, and $\Psi$, denoted by $X \sim$ $\operatorname{InvGa}(m, \mu, \theta, \Psi)$, if its p.d.f. is given by

$$
\frac{\operatorname{det}(X)^{-\mu-(m+1) / 2} \operatorname{etr}\left(-\Psi^{-1} X^{-1} / \theta\right)}{\operatorname{det}(\theta \Psi)^{\mu} \Gamma_{m}(\mu)}, \quad X>0,
$$

where $\mu>(m-1) / 2, \theta>0$, and $\Psi$ is a symmetric positive definite matrix of order $m$.

Definition 2. An $m \times m$ random symmetric positive definite matrix $U$ is said to have a matrix variate beta type 1 distribution with parameters $a(>(m-1) / 2)$ and $b(>(m-$ $1) / 2)$, denoted as $U \sim \mathrm{B} 1(m, a, b)$, if its p.d.f. is given by

$$
\frac{\operatorname{det}(U)^{a-(m+1) / 2} \operatorname{det}\left(I_{m}-U\right)^{b-(m+1) / 2}}{B_{m}(a, b)}, \quad 0<U<I_{m} .
$$

Definition 3. An $m \times m$ random symmetric positive definite matrix $V$ is said to have a matrix variate beta type 2 distribution with parameters $a(>(m-1) / 2)$ and $b(>(m-$ $1) / 2)$, denoted as $V \sim \mathrm{B} 2(m, a, b)$, if its p.d.f. is given by

$$
\frac{\operatorname{det}(V)^{a-(m+1) / 2} \operatorname{det}\left(I_{m}+V\right)^{-(a+b)}}{B_{m}(a, b)}, \quad V>0 .
$$

Note that if $U \sim \mathrm{B} 1(m, a, b)$, then $\left(I_{m}-U\right)^{-1} U \sim \mathrm{B} 2(m, a$, $b)$. Further, if $X_{1}$ and $X_{2}$ are independent, $X_{1} \sim \mathrm{Ga}\left(m, v_{1}, \theta\right)$ and $X_{2} \sim \mathrm{Ga}\left(m, v_{2}, \theta\right)$, then $\left(X_{1}+X_{2}\right)^{-1 / 2} X_{1}\left(X_{1}+X_{2}\right)^{-1 / 2} \sim$ $\mathrm{B} 1\left(m, v_{1}, v_{2}\right)$ and $X_{2}^{-1 / 2} X_{1} X_{2}^{-1 / 2} \sim \mathrm{B} 2\left(m, v_{1}, v_{2}\right)$.

We conclude this section by evaluating the normalizing constant $C(\nu, \alpha, \beta, \theta, \Omega)$ in (3). Since the density over its support set integrates to one, we have

$$
\begin{aligned}
& {[C(\nu, \alpha, \beta, \theta, \Omega)]^{-1}} \\
& \quad=\int_{X>0} \operatorname{det}(X)^{\nu-(m+1) / 2}{ }_{1} F_{1}\left(\alpha ; \beta ;-\frac{1}{\theta} \Omega^{-1} X\right) \mathrm{d} X .
\end{aligned}
$$

By rewriting ${ }_{1} F_{1}$ using Kummer's relation (24) and integrating $X$ by applying (21), we get

$$
\begin{aligned}
& {[C(\nu, \alpha, \beta, \theta, \Omega)]^{-1}} \\
& \quad=\Gamma_{m}(\nu) \operatorname{det}(\theta \Omega)_{2}{ }_{2} F_{1}\left(\nu, \beta-\alpha ; \beta ; I_{m}\right),
\end{aligned}
$$

where $\operatorname{Re}(\nu)>(m-1) / 2$. Finally, writing ${ }_{2} F_{1}\left(\nu, \beta-\alpha ; \beta ; I_{m}\right)$ in terms of multivariate gamma functions by using (23), we obtain

$$
\begin{aligned}
& {[C(\nu, \alpha, \beta, \theta, \Omega)]^{-1}} \\
& \quad=\int_{X>0} \operatorname{det}(X)^{\nu-(m+1) / 2}{ }_{1} F_{1}\left(\alpha ; \beta ;-\frac{1}{\theta} \Omega^{-1} X\right) \mathrm{d} X \\
& \quad=\frac{\operatorname{det}(\theta \Omega)^{\nu} \Gamma_{m}(\nu) \Gamma_{m}(\beta) \Gamma_{m}(\alpha-\nu)}{\Gamma_{m}(\alpha) \Gamma_{m}(\beta-\nu)}
\end{aligned}
$$

where $\operatorname{Re}(\beta-\nu)>(m-1) / 2, \operatorname{Re}(\alpha-\nu)>(m-1) / 2, \theta>0$, $\operatorname{Re}(\nu)>(m-1) / 2$, and $\Omega>0$.

\section{Properties}

In this section we study several properties of the confluent hypergeometric function kind 1 distribution defined in Section 1. For the sake of completeness, we first state the following results established in Gupta and Nagar [1].

(1) Let $X \sim \mathrm{CH}_{m}(\nu, \alpha, \beta, \theta, \Omega$, kind 1) and let $A$ be an $m \times m$ constant nonsingular matrix. Then, $A X A^{\prime} \sim$ $\mathrm{CH}_{m}\left(\nu, \alpha, \beta, \theta, A \Omega A^{\prime}\right.$, kind 1$)$.

(2) Let $X \sim \mathrm{CH}_{m}(\nu, \alpha, \beta, \theta$, kind 1) and let $H$ be an $m \times m$ orthogonal matrix whose elements are either constants or random variables distributed independent of $X$. Then, the distribution of $X$ is invariant under the transformation $X \rightarrow H X H^{\prime}$ if $H$ is a matrix of constants. Further, if $H$ is a random matrix, then $H$ and $H X H^{\prime}$ are independent.

(3) Let $X \sim \mathrm{CH}_{m}(\nu, \alpha, \beta, \theta, \Omega$, kind 1). Then, the cumulative distribution function (cdf) of $X$ is derived as

$$
\begin{aligned}
P(X \leq \Lambda)= & \frac{\Gamma_{m}(\alpha) \Gamma_{m}(\beta-\nu) \Gamma_{m}[(m+1) / 2]}{\Gamma_{m}[\nu+(m+1) / 2] \Gamma_{m}(\beta) \Gamma_{m}(\alpha-\nu)} \\
& \cdot \operatorname{det}(\theta \Omega)^{-v} \operatorname{det}(\Lambda)^{v} \\
& \cdot{ }_{2} F_{2}\left(\alpha, v ; \beta, v+\frac{m+1}{2} ;-\frac{1}{\theta} \Omega^{-1} \Lambda\right),
\end{aligned}
$$

where $\Lambda>0$.

(4) Let $X=\left(\begin{array}{ll}X_{11} & X_{12} \\ X_{21} & X_{22}\end{array}\right)$, where $X_{11}$ is a $q \times q$ matrix. Define $X_{11 \cdot 2}=X_{11}-X_{12} X_{22}^{-1} X_{21}$ and $X_{22 \cdot 1}=X_{22}-$ $X_{21} X_{11}^{-1} X_{12}$. If $X \sim \mathrm{CH}_{m}(\nu, \alpha, \beta, \theta$, kind 1), then (i) $X_{11}$ and $X_{22 \cdot 1}$ are independent, $X_{11} \sim \mathrm{CH}_{q}(\nu, \alpha-$ $(m-q) / 2, \beta-(m-q) / 2, \theta$, kind 1) and $X_{22 \cdot 1} \sim$ $\mathrm{CH}_{m-q}(\nu-q / 2, \alpha-q / 2, \beta-q / 2, \theta$, kind 1), and (ii) $X_{22}$ and $X_{11.2}$ are independent, $X_{22} \sim \mathrm{CH}_{m-q}(\nu, \alpha-$ $q / 2, \beta-q / 2, \theta$, kind 1$)$ and $X_{11.2} \sim \mathrm{CH}_{q}(\nu-(m-$ $q) / 2, \alpha-(m-q) / 2, \beta-(m-q) / 2, \theta$, kind 1$)$. 
(5) Let $A$ be a $q \times m$ constant matrix of rank $q(\leq$ $m)$. If $X \sim \mathrm{CH}_{m}(\nu, \alpha, \beta, \theta$, kind 1$)$, then $A X A^{\prime} \sim$ $\mathrm{CH}_{q}\left(\nu, \alpha-(m-q) / 2, \beta-(m-q) / 2, \theta, A A^{\prime}\right.$, kind 1$)$ and $\left(A X^{-1} A^{\prime}\right)^{-1} \sim \mathrm{CH}_{q}(\nu-(m-q) / 2, \alpha-(m-q) / 2, \beta-$ $(m-q) / 2, \theta,\left(A A^{\prime}\right)^{-1}$, kind 1$)$.

(6) Let $X \sim \mathrm{CH}_{m}(\nu, \alpha, \beta, \theta$, kind 1) and let a be a nonzero $m$-dimensional column vector of constants, then $\left(\mathbf{a}^{\prime} \mathbf{a}\right)^{-1}\left(\mathbf{a}^{\prime} X \mathbf{a}\right) \sim \mathrm{CH}(\nu, \alpha-(m-1) / 2, \beta-(m-$ 1) $/ 2, \theta$, kind 1) and $\mathbf{a}^{\prime} \mathbf{a}\left(\mathbf{a}^{\prime} X^{-1} \mathbf{a}\right)^{-1} \sim \mathrm{CH}(\nu-(m-$ $1) / 2, \alpha-(m-1) / 2, \beta-(m-1) / 2, \theta$, kind 1$)$. Further, if $\mathbf{y}$ is an $m$-dimensional random vector, independent of $X$, and $P(\mathbf{y} \neq \mathbf{0})=1$, then it follows that $\left(\mathbf{y}^{\prime} \mathbf{y}\right)^{-1}\left(\mathbf{y}^{\prime} X \mathbf{y}\right) \sim \mathrm{CH}(\nu, \alpha-(m-1) / 2, \beta-(m-$ 1) $/ 2, \theta$, kind 1) and $\mathbf{y}^{\prime} \mathbf{y}\left(\mathbf{y}^{\prime} X^{-1} \mathbf{y}\right)^{-1} \sim \mathrm{CH}(\nu-(m-$ $1) / 2, \alpha-(m-1) / 2, \beta-(m-1) / 2, \theta$, kind 1$)$.

It may also be mentioned here that properties (1)-(6) given above are modified forms of results given in Section 8.10 of Gupta and Nagar [1].

If the $m \times m$ random matrices $X_{1}$ and $X_{2}$ are independent, $X_{1} \sim \mathrm{CH}_{m}\left(\nu, a_{1}+\nu, 2 \nu, \theta\right.$, kind 1$)$ and $X_{2} \sim \mathrm{CH}_{m}\left(\nu, a_{2}+\right.$ $\nu, 2 \nu, \theta$, kind 1$), v=\gamma / 2+(m+1) / 4$, then Roux and van der Merwe [11] have shown that $X_{2}^{-1 / 2} X_{1} X_{2}^{-1 / 2}$ has matrix variate beta type 2 distributions with parameters $a_{2}$ and $a_{1}$.

The matrix variate confluent hypergeometric function kind 1 distribution can be derived as the distribution of the matrix ratio of independent gamma and beta matrices. It has been shown in Gupta and Nagar [1] that if $Y \sim \mathrm{Ga}(m, v, \theta)$ and $U \sim \mathrm{B} 1(m, a, b)$, then $U^{-1 / 2} Y U^{-1 / 2} \sim \mathrm{CH}_{m}(\nu, a+v, a+$ $b+v, \theta$, kind 1$)$.

The expected values of $X$ and $X^{-1}$, for $X$ $\mathrm{CH}_{m}(\nu, \alpha, \beta, \theta$, kind 1$)$, can easily be obtained from the above results. For any fixed $\mathbf{a} \in \mathbb{R}^{m}, \mathbf{a} \neq \mathbf{0}$,

$$
E\left[\frac{\mathbf{a}^{\prime} X \mathbf{a}}{\mathbf{a}^{\prime} \mathbf{a}}\right]=E\left(v_{1}\right),
$$

where $v_{1} \sim \mathrm{CH}(\nu, \alpha-(m-1) / 2, \beta-(m-1) / 2, \theta$, kind 1$)$, and

$$
E\left[\frac{\mathbf{a}^{\prime} X^{-1} \mathbf{a}}{\mathbf{a}^{\prime} \mathbf{a}}\right]=E\left(\frac{1}{v_{2}}\right),
$$

where $v_{2} \sim \mathrm{CH}(v-(m-1) / 2, \alpha-(m-1) / 2, \beta-(m-$ $1) / 2, \theta$, kind 1). Hence, for all $\mathbf{a} \in \mathbb{R}^{m}$,

$$
\begin{aligned}
\mathbf{a}^{\prime} E(X) \mathbf{a} & =\mathbf{a}^{\prime} \mathbf{a} E\left(v_{1}\right) \\
& =\frac{\nu \theta[\beta-\nu-(m+1) / 2]}{[\alpha-v-(m+1) / 2]} \mathbf{a}^{\prime} \mathbf{a}, \\
& \alpha-v>\frac{m+1}{2}, \beta-v>\frac{m+1}{2}, \\
\mathbf{a}^{\prime} E\left(X^{-1}\right) \mathbf{a} & =\mathbf{a}^{\prime} \mathbf{a} E\left(\frac{1}{v_{2}}\right) \\
& =\frac{\alpha-v}{\theta[\nu-(m+1) / 2](\beta-v)} \mathbf{a}^{\prime} \mathbf{a}, \\
& v>\frac{m+1}{2},
\end{aligned}
$$

which implies that

$$
\begin{aligned}
& E(X)=\frac{\nu \theta[\beta-\nu-(m+1) / 2]}{[\alpha-\nu-(m+1) / 2]} I_{m}, \\
& \alpha-\nu>\frac{m+1}{2}, \beta-v>\frac{m+1}{2}, \\
& E\left(X^{-1}\right)=\frac{\alpha-v}{\theta[\nu-(m+1) / 2](\beta-v)} I_{m}, \\
& v>\frac{m+1}{2} .
\end{aligned}
$$

The Laplace transform of the density of $X$, where $X \sim$ $\mathrm{CH}_{m}(\nu, \alpha, \beta, \theta$, kind 1$)$, is given by

$$
\begin{aligned}
& \frac{\theta^{-m \nu} \Gamma_{m}(\alpha) \Gamma_{m}(\beta-\nu)}{\Gamma_{m}(\nu) \Gamma_{m}(\beta) \Gamma_{m}(\alpha-\nu)} \int_{X>0} \operatorname{etr}\left(-Z X-\frac{X}{\theta}\right) \\
& \cdot \operatorname{det}(X)^{\nu-(m+1) / 2}{ }_{1} F_{1}\left(\beta-\alpha ; \beta ; \frac{X}{\theta}\right) \mathrm{d} X \\
& =\frac{\Gamma_{m}(\alpha) \Gamma_{m}(\beta-\nu)}{\Gamma_{m}(\beta) \Gamma_{m}(\alpha-\nu)} \operatorname{det}\left(I_{m}+\theta Z\right)^{-v}{ }_{2} F_{1}(\nu, \beta \\
& \left.-\alpha ; \beta ;\left(I_{m}+\theta Z\right)^{-1}\right), \quad \operatorname{Re}(Z)>0,
\end{aligned}
$$

where we have used (24) and (21). From the above expression, the Laplace transform of the density of $X$, where $X \sim$ $\mathrm{CH}_{m}(\nu, \alpha, \beta, \theta, \Omega$, kind 1$)$, is derived as

$$
\begin{aligned}
& \frac{\Gamma_{m}(\alpha) \Gamma_{m}(\beta-\nu)}{\Gamma_{m}(\beta) \Gamma_{m}(\alpha-\nu)} \operatorname{det}\left(I_{m}+\theta \Omega Z\right)^{-v} \\
& \quad \cdot{ }_{2} F_{1}\left(\nu, \beta-\alpha ; \beta ;\left(I_{m}+\theta \Omega Z\right)^{-1}\right), \quad \operatorname{Re}(Z)>0 .
\end{aligned}
$$

Theorem 4. Let $X \sim \mathrm{CH}_{m}(\nu, \alpha, \beta, \theta, \Omega$, kind 1$)$; then

$$
\begin{aligned}
& E\left[\operatorname{det}(X)^{h}\right] \\
& =\frac{\operatorname{det}(\theta \Omega)^{h} \Gamma_{m}(\beta-\nu) \Gamma_{m}(\nu+h) \Gamma_{m}(\alpha-\nu-h)}{\Gamma_{m}(\nu) \Gamma_{m}(\alpha-\nu) \Gamma_{m}(\beta-\nu-h)},
\end{aligned}
$$

where $\operatorname{Re}(h+v)>(m-1) / 2, \operatorname{Re}(h)<\alpha-v-(m-1) / 2$, and $\operatorname{Re}(h)<\beta-v-(m-1) / 2$.

Proof. From the density of $X$, we have

$$
\begin{aligned}
& E\left[\operatorname{det}(X)^{h}\right]=\frac{\operatorname{det}(\theta \Omega)^{-\nu} \Gamma_{m}(\alpha) \Gamma_{m}(\beta-\nu)}{\Gamma_{m}(\nu) \Gamma_{m}(\beta) \Gamma_{m}(\alpha-\nu)} \\
& \quad \cdot \int_{X>0} \operatorname{det}(X)^{\nu+h-(m+1) / 2}{ }_{1} F_{1}\left(\alpha ; \beta ;-\frac{1}{\theta} \Omega^{-1} X\right) d X .
\end{aligned}
$$

Now, evaluating the above integral by using (34), we get

$$
\begin{gathered}
E\left[\operatorname{det}(X)^{h}\right]=\frac{\operatorname{det}(\theta \Omega)^{-v} \Gamma_{m}(\alpha) \Gamma_{m}(\beta-\nu)}{\Gamma_{m}(\nu) \Gamma_{m}(\beta) \Gamma_{m}(\alpha-\nu)} \\
\cdot \frac{\operatorname{det}(\theta \Omega)^{\nu+h} \Gamma_{m}(\nu+h) \Gamma_{m}(\beta) \Gamma_{m}(\alpha-\nu-h)}{\Gamma_{m}(\alpha) \Gamma_{m}(\beta-\nu-h)},
\end{gathered}
$$


where $\operatorname{Re}(h+\nu)>(m-1) / 2, \operatorname{Re}(h)<\alpha-\nu-(m-1) / 2$, and $\operatorname{Re}(h)<\beta-\nu-(m-1) / 2$. Finally, simplifying the above expression, we get the desired result.

Corollary 5. Let $x \sim \mathrm{CH}(\nu, \alpha, \beta, \theta$, kind 1$)$; then

$$
E\left(x^{h}\right)=\frac{\theta^{h} \Gamma(\beta-v) \Gamma(\nu+h) \Gamma(\alpha-v-h)}{\Gamma(\nu) \Gamma(\alpha-\nu) \Gamma(\beta-v-h)},
$$

where $\operatorname{Re}(h+\nu)>0, \operatorname{Re}(h)<\alpha-\nu$, and $\operatorname{Re}(h)<\beta-\nu$.
Using (42) the mean and the variance of $\operatorname{det}(X)$ are derived as

$$
\begin{aligned}
& E[\operatorname{det}(X)]=\theta^{m} \operatorname{det}(\Omega) \\
& \cdot \prod_{j=1}^{m} \frac{[\nu-(j-1) / 2][\beta-\nu-(j+1) / 2]}{[\alpha-v-(j+1) / 2]},
\end{aligned}
$$

where $\beta>v+(m+1) / 2, \alpha>v+(m+1) / 2$, and

$$
\begin{aligned}
& \operatorname{Var}(\operatorname{det}(X))=\theta^{2 m} \operatorname{det}(\Omega)^{2} \prod_{j=1}^{m}\left[\frac { [ \nu - ( j - 1 ) / 2 ] [ \beta - v - ( j + 1 ) / 2 ] } { [ \alpha - \nu - ( j + 1 ) / 2 ] } \left\{\frac{[\nu-(j-3) / 2][\beta-\nu-(j+3) / 2]}{[\alpha-\nu-(j+3) / 2]}\right.\right. \\
& \left.\left.-\frac{[v-(j-1) / 2][\beta-v-(j+1) / 2]}{[\alpha-v-(j+1) / 2]}\right\}\right],
\end{aligned}
$$

where $\beta>v+(m+3) / 2$ and $\alpha>v+(m+3) / 2$. For $m \times m$ symmetric matrix $A, E\left[C_{\kappa}(A X)\right]$ is derived as

$$
\begin{gathered}
E\left[C_{\kappa}(A X)\right]=\frac{\operatorname{det}(\theta \Omega)^{-\nu} \Gamma_{m}(\alpha) \Gamma_{m}(\beta-\nu)}{\Gamma_{m}(\nu) \Gamma_{m}(\beta) \Gamma_{m}(\alpha-\nu)} \\
\cdot \int_{X>0} C_{\kappa}(A X) \operatorname{det}(X)^{\nu-(m+1) / 2} \\
\cdot{ }_{1} F_{1}\left(\alpha ; \beta ;-\frac{1}{\theta} \Omega^{-1} X\right) \mathrm{d} X .
\end{gathered}
$$

Replacing ${ }_{1} F_{1}\left(\alpha ; \beta ;-\Omega^{-1} X / \theta\right)$ by its integral representation, namely,

$$
\begin{aligned}
& { }_{1} F_{1}\left(\alpha ; \beta ;-\frac{1}{\theta} \Omega^{-1} X\right)=\frac{\Gamma_{m}(\beta)}{\Gamma_{m}(\alpha) \Gamma_{m}(\beta-\alpha)} \\
& \cdot \int_{0}^{I_{m}} \operatorname{etr}\left(-\frac{1}{\theta} \Omega^{-1 / 2} X \Omega^{-1 / 2} Y\right) \operatorname{det}(Y)^{\alpha-(m+1) / 2} \\
& \cdot \operatorname{det}\left(I_{m}-Y\right)^{\beta-\alpha-(m+1) / 2} \mathrm{~d} Y,
\end{aligned}
$$

where $\operatorname{Re}(\beta-\alpha)>(m-1) / 2$ and $\operatorname{Re}(\alpha)>(m-1) / 2$, one obtains

$$
\begin{aligned}
& E\left[C_{\kappa}(A X)\right]=\frac{\operatorname{det}(\theta \Omega)^{-\nu} \Gamma_{m}(\beta-\nu)}{\Gamma_{m}(\nu) \Gamma_{m}(\beta-\alpha) \Gamma_{m}(\alpha-\nu)} \\
& \cdot \int_{0}^{I_{m}} \operatorname{det}(Y)^{\alpha-(m+1) / 2} \operatorname{det}\left(I_{m}-Y\right)^{\beta-\alpha-(m+1) / 2} \\
& \cdot \int_{X>0} C_{\kappa}(A X) \operatorname{det}(X)^{\nu-(m+1) / 2} \\
& \cdot \operatorname{etr}\left(-\frac{1}{\theta} \Omega^{-1 / 2} X \Omega^{-1 / 2} Y\right) d X d Y .
\end{aligned}
$$

Now, evaluating the above integral by using (19), we obtain

$$
\begin{aligned}
& E\left[C_{\kappa}(A X)\right]=\frac{\theta^{k} \Gamma_{m}(\beta-\nu)(\nu)_{\kappa}}{\Gamma_{m}(\beta-\alpha) \Gamma_{m}(\alpha-\nu)} \\
& \quad \cdot \int_{0}^{I_{m}} \operatorname{det}(Y)^{\alpha-\nu-(m+1) / 2} \operatorname{det}\left(I_{m}-Y\right)^{\beta-\alpha-(m+1) / 2} \\
& \cdot C_{\kappa}\left(\Omega^{1 / 2} A \Omega^{1 / 2} Y^{-1}\right) d Y .
\end{aligned}
$$

Finally, evaluating the integral involving $Y$ by using (Khatrzi [12])

$$
\begin{aligned}
& \int_{0}^{I_{m}} \operatorname{det}(R)^{a-(m+1) / 2} \operatorname{det}\left(I_{m}-R\right)^{b-(m+1) / 2} \\
& \cdot C_{\kappa}\left(X R^{-1}\right) \mathrm{d} R \\
& =\frac{(-a-b+(m+1) / 2)_{\kappa} \Gamma_{m}(a) \Gamma_{m}(b)}{(-a+(m+1) / 2)_{\kappa} \Gamma_{m}(a+b)} C_{\kappa}(X), \\
& \operatorname{Re}(a)>\frac{m-1}{2}+k_{1}, \operatorname{Re}(b)>\frac{m-1}{2},
\end{aligned}
$$

we get

$$
\begin{aligned}
E\left[C_{\kappa}(A X)\right] & \\
& =\frac{\theta^{k}(\nu-\beta+(m+1) / 2)_{\kappa}(\nu)_{\kappa}}{(\nu-\alpha+(m+1) / 2)_{\kappa}} C_{\kappa}(\Omega A),
\end{aligned}
$$

where $\operatorname{Re}(\alpha-\nu)>(m-1) / 2+k_{1}$ and $\operatorname{Re}(\beta-\nu)>(m-1) / 2+k_{1}$.

Proceeding similarly and using the result (Khatrzi [12])

$$
\begin{aligned}
& \int_{R>0} \operatorname{etr}(-X R) \operatorname{det}(R)^{a-(m+1) / 2} C_{\kappa}\left(R^{-1} S\right) \mathrm{d} R \\
&= \frac{(-1)^{k} \Gamma_{m}(a)}{(-a+(m+1) / 2)_{\kappa}} \operatorname{det}(X)^{-a} C_{\kappa}(X S), \\
& \operatorname{Re}(a)>\frac{m-1}{2}+k_{1},
\end{aligned}
$$


the expected value of $C_{\kappa}\left(A X^{-1}\right)$ is derived as

$$
\begin{aligned}
& E\left[C_{\kappa}\left(A X^{-1}\right)\right]=\frac{\operatorname{det}(\theta \Omega)^{-\nu} \Gamma_{m}(\beta-\nu)}{\Gamma_{m}(\nu) \Gamma_{m}(\beta-\alpha) \Gamma_{m}(\alpha-\nu)} \\
& \cdot \int_{0}^{I_{m}} \operatorname{det}(Y)^{\alpha-(m+1) / 2} \operatorname{det}\left(I_{m}-Y\right)^{\beta-\alpha-(m+1) / 2} \\
& \cdot \int_{X>0} C_{\kappa}\left(A X^{-1}\right) \operatorname{det}(X)^{\nu-(m+1) / 2} \\
& \cdot \operatorname{etr}\left(-\frac{1}{\theta} \Omega^{-1 / 2} X \Omega^{-1 / 2} Y\right) \mathrm{d} X \mathrm{~d} Y \\
& =\frac{(-1)^{k} \theta^{-k} \Gamma_{m}(\beta-\nu)}{\Gamma_{m}(\beta-\alpha) \Gamma_{m}(\alpha-\nu)(-\nu+(m+1) / 2)_{\kappa}} \\
& \cdot \int_{0}^{I_{m}} \operatorname{det}(Y)^{\alpha-\nu-(m+1) / 2} \operatorname{det}\left(I_{m}-Y\right)^{\beta-\alpha-(m+1) / 2} \\
& \cdot C_{\kappa}\left(\Omega^{-1 / 2} A \Omega^{-1 / 2} Y\right) \mathrm{d} Y,
\end{aligned}
$$

where $\operatorname{Re}(\nu)>(m-1) / 2+k_{1}$. Finally, evaluating the above integral using (20) we obtain

$$
\begin{aligned}
& E\left[C_{\kappa}\left(A X^{-1}\right)\right] \\
& =\frac{(-1)^{k} \theta^{-k}(\alpha-\nu)_{\kappa}}{(\beta-\nu)_{\kappa}(-\nu+(m+1) / 2)_{\kappa}} C_{\kappa}\left(\Omega^{-1} A\right), \\
& \operatorname{Re}(\nu)>\frac{m-1}{2}+k_{1} .
\end{aligned}
$$

In the next theorem, we derive the confluent hypergeometric function kind 1 distribution using independent beta and gamma matrices.

Theorem 6. Let $X_{1}$ and $X_{2}$ be independent, $X_{1} \sim \mathrm{Ga}(m, v, \theta)$ and $X_{2} \sim \mathrm{B} 1(m, a, b)$. Then, $X_{2}^{-1 / 2} X_{1} X_{2}^{-1 / 2} \sim \mathrm{CH}_{m}(\nu, a+\nu, a+$ $b+\nu, \theta$, kind 1$)$.

\section{Proof. See Gupta and Nagar [1].}

Theorem 7. Let $X_{1}$ and $X_{2}$ be independent, $X_{1} \sim \mathrm{Ga}(m, v, \theta)$ and $X_{2} \sim \mathrm{B} 1(m, a, b)$. Then, $X_{1}^{1 / 2} X_{2}^{-1} X_{1}^{1 / 2} \sim \mathrm{CH}_{m}(v, a+v, a+$ $b+v, \theta$, kind 1$)$

Proof. The result follows from Theorem 6 and the fact that $X_{2}^{-1 / 2} X_{1} X_{2}^{-1 / 2}$ and $X_{1}^{1 / 2} X_{2}^{-1} X_{1}^{1 / 2}$ have same eigenvalues, and the matrix variate confluent hypergeometric function kind 1 distribution is orthogonally invariant.

Theorem 8. Let $X_{1}$ and $X_{2}$ be independent, $X_{1} \sim \mathrm{Ga}(m, v, \theta)$ and $X_{2} \sim \mathrm{B} 1(m, a, b)$. Then, $\left(I_{m}-X_{2}\right)^{-1 / 2} X_{1}\left(I_{m}-X_{2}\right)^{-1 / 2} \sim$ $\mathrm{CH}_{m}(\nu, b+\nu, a+b+\nu, \theta$, kind 1$)$.

Proof. Noting that $I_{m}-X_{2} \sim \mathrm{B} 1(m, b, a)$ and using Theorem 6 we get the result.

Theorem 9. Let $X_{1}$ and $X_{2}$ be independent, $X_{1} \sim \mathrm{Ga}(m, v, \theta)$ and $X_{2} \sim \mathrm{B} 2(m, a, b)$. Then, $\left(I_{m}+X_{2}\right)^{1 / 2} X_{1}\left(I_{m}+X_{2}\right)^{1 / 2} \sim$ $\mathrm{CH}_{m}(\nu, b+v, a+b+v, \theta$, kind 1$)$.
Proof. The desired result is obtained by observing that $\left(I_{m}+\right.$ $\left.X_{2}\right)^{-1} \sim \mathrm{B} 1(m, b, a)$ and using Theorem 6 .

Theorem 10. Let $X_{1}$ and $X_{2}$ be independent, $X_{1} \sim \mathrm{Ga}(m, \nu, \theta)$ and $X_{2} \sim \mathrm{B} 2(m, a, b)$. Then, $\left(I_{m}+X_{2}^{-1}\right)^{1 / 2} X_{1}\left(I_{m}+X_{2}^{-1}\right)^{1 / 2} \sim$ $\mathrm{CH}_{m}(\nu, a+v, a+b+\nu, \theta$, kind 1$)$.

Proof. Noting that $\left(I_{m}+X_{2}^{-1}\right)^{-1} \sim \mathrm{B} 1(m, a, b)$ and using Theorem 6 we get the result.

Theorem 11. Let $X_{1}$ and $X_{2}$ be independent, $X_{1} \sim \mathrm{Ga}\left(m, v_{1}\right.$, $\theta)$ and $X_{2} \sim \mathrm{Ga}\left(m, v_{2}, \theta\right)$. Then, $\left(X_{1}+X_{2}\right) X_{1}^{-1}\left(X_{1}+X_{2}\right) \sim$ $\mathrm{CH}_{m}\left(\nu_{1}+v_{2}, 2 \nu_{1}+v_{2}, 2 \nu_{1}+2 v_{2}, \theta\right.$, kind 1$)$.

Proof. It is well known that $X_{1}+X_{2}$ and $\left(X_{1}+X_{2}\right)^{-1 / 2} X_{1}\left(X_{1}+\right.$ $\left.X_{2}\right)^{-1 / 2}$ are independent, $X_{1}+X_{2} \sim \mathrm{Ga}\left(m, v_{1}+v_{2}, \theta\right)$ and $\left(X_{1}+X_{2}\right)^{-1 / 2} X_{1}\left(X_{1}+X_{2}\right)^{-1 / 2} \sim \mathrm{B} 1\left(m, v_{1}, v_{2}\right)$. Therefore, using Theorem $7,\left(X_{1}+X_{2}\right) X_{1}^{-1}\left(X_{1}+X_{2}\right) \sim \mathrm{CH}_{m}\left(\nu_{1}+v_{2}, 2 v_{1}+\right.$ $v_{2}, 2 v_{1}+2 v_{2}, \theta$, kind 1$)$.

Theorem 12. Let $X_{1}$ and $X_{2}$ be independent, $X_{1} \sim$ $\mathrm{Ga}\left(m, v_{1}, \theta\right)$ and $X_{2} \sim \mathrm{Ga}\left(m, v_{2}, \theta\right)$. Then, $\left(X_{1}+X_{2}\right) X_{2}^{-1}\left(X_{1}+\right.$ $\left.X_{2}\right) \sim \mathrm{CH}_{m}\left(\nu_{1}+v_{2}, \nu_{1}+2 v_{2}, 2 v_{1}+2 v_{2}, \theta\right.$, kind 1$)$.

Proof. The proof is similar to the proof of Theorem 11.

\section{Distributions of Sum and Quotients}

In statistical distribution theory it is well known that if $X_{1}$ and $X_{2}$ are independent, $X_{1} \sim \operatorname{Ga}\left(m, v_{1}, \theta\right)$ and $X_{2} \sim$ $\mathrm{Ga}\left(m, v_{2}, \theta\right)$, then $X_{2}^{-1 / 2} X_{1} X_{2}^{-1 / 2} \sim \mathrm{B} 2\left(m, v_{1}, v_{2}\right),\left(X_{1}+\right.$ $\left.X_{2}\right)^{-1 / 2} X_{1}\left(X_{1}+X_{2}\right)^{-1 / 2} \sim \mathrm{B} 1\left(m, v_{1}, v_{2}\right)$, and $X_{1}+X_{2} \sim$ $\mathrm{Ga}\left(m, v_{1}+v_{2}\right)$. In this section we derive similar results when $X_{1}$ and $X_{2}$ are independent confluent hypergeometric function kind 1 and gamma matrices, respectively.

Theorem 13. Let $X_{1}$ and $X_{2}$ be independent, $X_{1} \sim \mathrm{CH}_{m}\left(\nu_{1}\right.$, $\alpha_{1}, \beta_{1}, \theta$, kind 1$)$ and $X_{2} \sim \mathrm{Ga}\left(m, v_{2}, \theta\right)$. Then, the p.d.f. of $Z=$ $X_{2}^{-1 / 2} X_{1} X_{2}^{-1 / 2}$ is given by

$$
\begin{array}{r}
\frac{\Gamma_{m}\left(\alpha_{1}\right) \Gamma_{m}\left(\beta_{1}-v_{1}\right) \Gamma_{m}\left(\nu_{1}+v_{2}\right)}{\Gamma_{m}\left(\beta_{1}\right) \Gamma_{m}\left(\alpha_{1}-v_{1}\right) \Gamma_{m}\left(v_{1}\right) \Gamma_{m}\left(\nu_{2}\right)} \frac{\operatorname{det}(Z)^{\nu_{1}-(m+1) / 2}}{\operatorname{det}\left(I_{m}+Z\right)^{\nu_{1}+v_{2}}} \\
\cdot{ }_{2} F_{1}\left(\beta_{1}-\alpha_{1}, \nu_{1}+v_{2} ; \beta_{1} ;\left(I_{m}+Z\right)^{-1} Z\right), \\
Z>0 .
\end{array}
$$

Proof. Using the independence, the joint p.d.f. of $X_{1}$ and $X_{2}$ is given by

$$
\begin{aligned}
& K \operatorname{det}\left(X_{1}\right)^{\nu_{1}-(m+1) / 2} \operatorname{det}\left(X_{2}\right)^{\nu_{2}-(m+1) / 2} \\
& \cdot \operatorname{etr}\left[-\frac{1}{\theta}\left(X_{1}+X_{2}\right)\right]{ }_{1} F_{1}\left(\beta_{1}-\alpha_{1} ; \beta_{1} ; \frac{1}{\theta} X_{1}\right), \\
& X_{1}>0, X_{2}>0,
\end{aligned}
$$


where

$$
K=\frac{\theta^{-m\left(v_{1}+v_{2}\right)} \Gamma_{m}\left(\alpha_{1}\right) \Gamma_{m}\left(\beta_{1}-v_{1}\right)}{\Gamma_{m}\left(\nu_{1}\right) \Gamma_{m}\left(\nu_{2}\right) \Gamma_{m}\left(\beta_{1}\right) \Gamma_{m}\left(\alpha_{1}-v_{1}\right)}
$$

Making the transformation $Z=X_{2}^{-1 / 2} X_{1} X_{2}^{-1 / 2}, X_{2}=X_{2}$, with the Jacobian $J\left(X_{1}, X_{2} \rightarrow Z, X_{2}\right)=\operatorname{det}\left(X_{2}\right)^{(m+1) / 2}$ we obtain the joint p.d.f. of $Z$ and $X_{2}$ as

$$
\begin{array}{r}
K \operatorname{det}(Z)^{v_{1}-(m+1) / 2} \operatorname{det}\left(X_{2}\right)^{\nu_{1}+v_{2}-(m+1) / 2} \\
\cdot \exp \left[-\frac{1}{\theta}\left(I_{m}+Z\right) X_{2}\right]{ }_{1} F_{1}\left(\beta_{1}-\alpha_{1} ; \beta_{1} ; \frac{1}{\theta} Z X_{2}\right), \\
Z>0, X_{2}>0 .
\end{array}
$$

Now, integrating $X_{2}$ in (60) by applying (21) and substituting for $K$, we obtain the desired result.

Corollary 14. Let $X_{1}$ and $X_{2}$ be independent, $X_{1} \sim$ $\mathrm{CH}_{m}\left(\nu_{1}, \alpha_{1}, \beta_{1}, \theta\right.$, kind 1) and $X_{2} \sim \mathrm{Ga}\left(m, \nu_{2}, \theta\right)$. Then, the p.d.f. of $Z_{1}=X_{2}^{1 / 2} X_{1}^{-1} X_{2}^{1 / 2}$ is given by

$$
\begin{aligned}
& \frac{\Gamma_{m}\left(\alpha_{1}\right) \Gamma_{m}\left(\beta_{1}-v_{1}\right) \Gamma_{m}\left(v_{1}+v_{2}\right)}{\Gamma_{m}\left(\beta_{1}\right) \Gamma_{m}\left(\alpha_{1}-v_{1}\right) \Gamma_{m}\left(\nu_{1}\right) \Gamma_{m}\left(v_{2}\right)} \\
& \quad \cdot \frac{\operatorname{det}\left(Z_{1}\right)^{\nu_{2}-(m+1) / 2}}{\operatorname{det}\left(I_{m}+Z_{1}\right)^{\nu_{1}+v_{2}}} \\
& \quad \cdot{ }_{2} F_{1}\left(\beta_{1}-\alpha_{1}, v_{1}+v_{2} ; \beta_{1} ;\left(I_{m}+Z_{1}\right)^{-1}\right), \quad Z_{1}>0 .
\end{aligned}
$$

Corollary 15. Let $X_{1}$ and $X_{2}$ be independent, $X_{1} \sim \mathrm{Ga}(m$, $\left.\nu_{1}, \theta\right)$ and $X_{2} \sim \mathrm{CH}_{m}\left(\nu_{2}, \alpha_{2}, \beta_{2}, \theta\right.$, kind 1$)$. Then, the p.d.f. of $Z_{3}=X_{2}^{-1 / 2} X_{1} X_{2}^{-1 / 2}$ is given by

$$
\begin{aligned}
& \frac{\Gamma_{m}\left(\alpha_{2}\right) \Gamma_{m}\left(\beta_{2}-v_{2}\right) \Gamma_{m}\left(v_{1}+v_{2}\right)}{\Gamma_{m}\left(\beta_{2}\right) \Gamma_{m}\left(\alpha_{2}-v_{2}\right) \Gamma_{m}\left(v_{1}\right) \Gamma_{m}\left(v_{2}\right)} \\
& \quad \cdot \frac{\operatorname{det}\left(Z_{3}\right)^{\nu_{1}-(m+1) / 2}}{\operatorname{det}\left(I_{m}+Z_{3}\right)^{\nu_{1}+v_{2}}} \\
& \quad \cdot{ }_{2} F_{1}\left(\beta_{2}-\alpha_{2}, v_{1}+v_{2} ; \beta_{2} ;\left(I_{m}+Z_{3}\right)^{-1}\right), \quad Z_{3}>0 .
\end{aligned}
$$

Proof. Interchanging subscripts 1 and 2 in Corollary 14, the p.d.f. of $Z_{2}=X_{1}^{1 / 2} X_{2}^{-1} X_{1}^{1 / 2}$ is given by

$$
\begin{aligned}
& \frac{\Gamma_{m}\left(\alpha_{2}\right) \Gamma_{m}\left(\beta_{2}-v_{2}\right) \Gamma_{m}\left(v_{1}+v_{2}\right)}{\Gamma_{m}\left(\beta_{2}\right) \Gamma_{m}\left(\alpha_{2}-v_{2}\right) \Gamma_{m}\left(\nu_{1}\right) \Gamma_{m}\left(v_{2}\right)} \\
& \quad \cdot \frac{\operatorname{det}\left(Z_{2}\right)^{\nu_{1}-(m+1) / 2}}{\operatorname{det}\left(I_{m}+Z_{2}\right)^{\nu_{1}+v_{2}}} \\
& \quad \cdot{ }_{2} F_{1}\left(\beta_{2}-\alpha_{2}, v_{1}+v_{2} ; \beta_{2} ;\left(I_{m}+Z_{2}\right)^{-1}\right), \quad Z_{2}>0,
\end{aligned}
$$

where $X_{1} \sim \mathrm{Ga}\left(m, v_{1}, \theta\right)$ and $X_{2} \sim \mathrm{CH}_{m}\left(\nu_{2}, \alpha_{2}, \beta_{2}, \theta\right.$, kind 1). Now, the result follows from the fact that $Z_{2}=$ $X_{1}^{1 / 2} X_{2}^{-1} X_{1}^{1 / 2}$ and $Z_{3}=X_{2}^{-1 / 2} X_{1} X_{2}^{-1 / 2}$ have the same eigenvalues, and the distribution $Z_{2}$ is orthogonally invariant.
Corollary 16. Let $X_{1}$ and $X_{2}$ be independent, $X_{1} \sim$ $\mathrm{CH}_{m}\left(\nu_{1}, \alpha_{1}, v_{1}+v_{2}, \theta\right.$, kind 1) and $X_{2} \sim \mathrm{Ga}\left(m, v_{2}, \theta\right)$. Then, $X_{2}^{-1 / 2} X_{1} X_{2}^{-1 / 2} \sim \mathrm{B} 2\left(m, v_{1}, \alpha_{1}-v_{1}\right)$.

Corollary 17. Let the random matrices $X_{1}$ and $X_{2}$ be independent, $X_{1} \sim \mathrm{Ga}\left(m, v_{1}, \theta\right)$ and $X_{2} \sim \mathrm{CH}_{m}\left(\nu_{2}, \alpha_{2}, v_{2}+v_{1}\right.$, $\theta$, kind 1). Then, $X_{2}^{-1 / 2} X_{1} X_{2}^{-1 / 2} \sim \mathrm{B} 2\left(m, \alpha_{2}-v_{2}, v_{2}\right)$.

Corollary 18. Let $X_{1}$ and $X_{2}$ be independent, $X_{1} \sim \mathrm{Ga}(m$, $\left.\nu_{1}, \theta\right)$ and $X_{2} \sim \mathrm{Ga}\left(m, v_{2}, \theta\right)$. Then, $X_{2}^{-1 / 2} X_{1} X_{2}^{-1 / 2} \sim \mathrm{B} 2(m$, $\left.v_{1}, v_{2}\right)$.

Theorem 19. Let $X_{1}, X_{2}$, and $X_{3}$ be independent, $X_{1} \sim$ $\mathrm{Ga}(m, \mu, \theta), X_{2} \sim \mathrm{B} 1(m, a, b)$, and $X_{3} \sim \mathrm{Ga}(m, v, \theta)$. Then, the p.d.f. of $Z=X_{3}^{-1 / 2} X_{2}^{-1 / 2} X_{1} X_{2}^{-1 / 2} X_{3}^{-1 / 2}$ is given by

$$
\begin{aligned}
& \frac{\Gamma_{m}(a+\mu) \Gamma_{m}(a+b) \Gamma_{m}(\mu+\nu)}{\Gamma_{m}(a+b+\mu) \Gamma_{m}(a) \Gamma_{m}(\mu) \Gamma_{m}(\nu)} \frac{\operatorname{det}(Z)^{\mu-(m+1) / 2}}{\operatorname{det}\left(I_{m}+Z\right)^{\mu+\nu}} \\
& \quad{ }_{2} F_{1}\left(b, \mu+v ; a+b+\mu ;\left(I_{m}+Z\right)^{-1} Z\right), \quad Z>0 .
\end{aligned}
$$

Proof. Using the independence of $X_{1}$ and $X_{2}$ and Theorem 6, $X_{2}^{-1 / 2} X_{1} X_{2}^{-1 / 2} \sim \mathrm{CH}_{m}(\mu, a+\mu, a+b+\mu$, kind 1). Further, using independence of $X_{2}^{-1 / 2} X_{1} X_{2}^{-1 / 2}$ and $X_{3}$ and Theorem 13, we obtain the desired result.

Corollary 20. Let $X_{1}, X_{2}$, and $X_{3}$ be independent, $X_{1} \sim$ $\mathrm{Ga}(m, \mu, \theta), X_{2} \sim \mathrm{B} 1(m, a, b)$, and $X_{3} \sim \mathrm{Ga}(m, a+b, \theta)$. Then, $X_{3}^{-1 / 2} X_{2}^{-1 / 2} X_{1} X_{2}^{-1 / 2} X_{3}^{-1 / 2} \sim \mathrm{B} 2(m, \mu, a)$.

Proof. For $v=a+b$, the p.d.f. of $Z=X_{3}^{-1 / 2} X_{2}^{-1 / 2} X_{1} X_{2}^{-1 / 2}$. $X_{3}^{-1 / 2}$ given in the above theorem slides to

$$
\begin{aligned}
& \frac{\Gamma_{m}(a+\mu)}{\Gamma_{m}(a) \Gamma_{m}(\mu)} \frac{\operatorname{det}(Z)^{\mu-(m+1) / 2}}{\operatorname{det}\left(I_{m}+Z\right)^{\mu+a+b}} \\
& \quad \cdot{ }_{2} F_{1}\left(b, \mu+a+b ; a+b+\mu ;\left(I_{m}+Z\right)^{-1} Z\right),
\end{aligned}
$$

$Z>0$.

Now, simplifying the Gauss hypergeometric function as

$$
\begin{aligned}
& { }_{2} F_{1}\left(b, \mu+a+b ; a+b+\mu ;\left(I_{m}+Z\right)^{-1} Z\right) \\
& \quad={ }_{1} F_{0}\left(b ;\left(I_{m}+Z\right)^{-1} Z\right) \\
& \quad=\operatorname{det}\left(I_{m}-\left(I_{m}+Z\right)^{-1} Z\right)^{-b}=\operatorname{det}\left(I_{m}+Z\right)^{b},
\end{aligned}
$$

where we have used (16), the desired result is obtained.

Corollary 21. Let $V$ and $U$ be independent, $V \sim \mathrm{B} 2(m, \mu, \nu)$ and $U \sim \mathrm{B} 1(m, a, b)$. Then, the p.d.f. of $Z=U^{-1 / 2} V U^{-1 / 2}$ is given by

$$
\begin{aligned}
& \frac{\Gamma_{m}(a+\mu) \Gamma_{m}(a+b) \Gamma_{m}(\mu+\nu)}{\Gamma_{m}(a+b+\mu) \Gamma_{m}(a) \Gamma_{m}(\mu) \Gamma_{m}(\nu)} \frac{\operatorname{det}(Z)^{\mu-(m+1) / 2}}{\operatorname{det}\left(I_{m}+Z\right)^{\mu+\nu}} \\
& \quad \cdot{ }_{2} F_{1}\left(b, \mu+v ; a+b+\mu ;\left(I_{m}+Z\right)^{-1} Z\right), \quad Z>0 .
\end{aligned}
$$

Further, if $v=a+b$, then $U^{-1 / 2} V U^{-1 / 2} \sim \mathrm{B} 2(m, \mu, a)$. 
Proof. Observe that $Z=X_{3}^{-1 / 2} X_{2}^{-1 / 2} X_{1} X_{2}^{-1 / 2} X_{3}^{-1 / 2}$ and $X_{2}^{-1 / 2} X_{3}^{-1 / 2} X_{1} X_{3}^{-1 / 2} X_{2}^{-1 / 2}$ have same eigenvalues and the distribution of $Z$ is orthogonally invariant. Therefore, the random matrices $X_{3}^{-1 / 2} X_{2}^{-1 / 2} X_{1} X_{2}^{-1 / 2} X_{3}^{-1 / 2}$ and $X_{2}^{-1 / 2} X_{3}^{-1 / 2} X_{1} X_{3}^{-1 / 2} X_{2}^{-1 / 2}$ have identical distribution. Now, setting $V=X_{3}^{-1 / 2} X_{1} X_{3}^{-1 / 2}$ and $U=X_{2}$, where $X_{3}^{-1 / 2} X_{1} X_{3}^{-1 / 2} \sim \mathrm{B} 2(m, \mu, v)$ and $X_{2} \sim \mathrm{B} 1(m, a, b)$, we observe that $X_{3}^{-1 / 2} X_{2}^{-1 / 2} X_{1} X_{2}^{-1 / 2} X_{3}^{-1 / 2}$ and $U^{-1 / 2} V U^{-1 / 2}$ have identical distribution.

Theorem 22. Let $X_{1}$ and $X_{2}$ be independent, $X_{1}$ $\mathrm{CH}_{m}\left(\nu_{1}, \alpha_{1}, \beta_{1}, \theta\right.$, kind 1) and $X_{2} \sim \mathrm{Ga}\left(m, v_{2}, \theta\right)$. Then, the p.d.f. of $R=\left(X_{1}+X_{2}\right)^{-1 / 2} X_{1}\left(X_{1}+X_{2}\right)^{-1 / 2}$ is given by

$$
\begin{aligned}
& \frac{\Gamma_{m}\left(\alpha_{1}\right) \Gamma_{m}\left(\beta_{1}-v_{1}\right) \Gamma_{m}\left(\nu_{1}+v_{2}\right)}{\Gamma_{m}\left(\beta_{1}\right) \Gamma_{m}\left(\alpha_{1}-v_{1}\right) \Gamma_{m}\left(\nu_{1}\right) \Gamma_{m}\left(\nu_{2}\right)} \operatorname{det}(R)^{v_{1}-(m+1) / 2} \\
& \cdot \operatorname{det}\left(I_{m}-R\right)^{\nu_{2}-(m+1) / 2} \\
& \quad \cdot{ }_{2} F_{1}\left(\beta_{1}-\alpha_{1}, v_{1}+v_{2} ; \beta_{1} ; R\right), \quad 0<R<I_{m}
\end{aligned}
$$

and the p.d.f. of $S=X_{1}+X_{2}$ is given by

$$
\begin{gathered}
\frac{\theta^{-m\left(\nu_{1}+v_{2}\right)} \Gamma_{m}\left(\alpha_{1}\right) \Gamma_{m}\left(\beta_{1}-v_{1}\right)}{\Gamma_{m}\left(\beta_{1}\right) \Gamma_{m}\left(\alpha_{1}-v_{1}\right) \Gamma_{m}\left(\nu_{1}+v_{2}\right)} \operatorname{det}(S)^{\nu_{1}+\nu_{2}-(m+1) / 2} \\
\cdot \operatorname{etr}\left(-\frac{1}{\theta} S\right)_{2} F_{2}\left(\nu_{1}, \beta_{1}-\alpha_{1} ; v_{1}+v_{2}, \beta_{1} ; \frac{1}{\theta} S\right),
\end{gathered}
$$$$
S>0 .
$$

Proof. Substituting $R=\left(X_{1}+X_{2}\right)^{-1 / 2} X_{1}\left(X_{1}+X_{2}\right)^{-1 / 2}$ and $S=$ $X_{1}+X_{2}$ with the Jacobian $J\left(X_{1}, X_{2} \rightarrow R, S\right)=\operatorname{det}(S)^{(m+1) / 2}$ in (58) we obtain the joint p.d.f. of $R$ and $S$ as

$$
\begin{aligned}
& K \operatorname{det}(R)^{\nu_{1}-(m+1) / 2} \operatorname{det}\left(I_{m}-R\right)^{\nu_{2}-(m+1) / 2} \\
& \cdot \operatorname{det}(S)^{\nu_{1}+v_{2}-(m+1) / 2} \operatorname{etr}\left(-\frac{1}{\theta} S\right) \\
& \quad \cdot{ }_{1} F_{1}\left(\beta_{1}-\alpha_{1} ; \beta_{1} ; \frac{1}{\theta} R S\right), \quad 0<R<I_{m}, S>0 .
\end{aligned}
$$

Now, integration of $S$ by using (21) yields the density of $R$. The marginal density of $S$ is obtained by integrating $R$ by using (22).

It may be remarked here that the density of $R$ given in the above theorem can also be obtained from the density of $Z=X_{2}^{-1 / 2} X_{1} X_{2}^{-1 / 2}$ derived in Theorem 13 by making the transformation $R=\left(I_{m}+Z\right)^{-1} Z$.

Corollary 23. Let $X_{1}$ and $X_{2}$ be independent random matrices, $X_{1} \sim \mathrm{Ga}\left(m, v_{1}, \theta\right)$ and $X_{2} \sim \mathrm{CH}_{m}\left(\nu_{2}, \alpha_{2}, \beta_{2}, \theta\right.$, kind 1$)$.
Then, the p.d.f. of $R_{1}=\left(X_{1}+X_{2}\right)^{-1 / 2} X_{1}\left(X_{1}+X_{2}\right)^{-1 / 2}$ is given by

$$
\begin{aligned}
& \frac{\Gamma_{m}\left(\alpha_{2}\right) \Gamma_{m}\left(\beta_{2}-v_{2}\right) \Gamma_{m}\left(v_{1}+v_{2}\right)}{\Gamma_{m}\left(\beta_{2}\right) \Gamma_{m}\left(\alpha_{2}-v_{2}\right) \Gamma_{m}\left(\nu_{1}\right) \Gamma_{m}\left(v_{2}\right)} \operatorname{det}\left(R_{1}\right)^{\nu_{1}-(m+1) / 2} \\
& \quad \cdot \operatorname{det}\left(I_{m}-R_{1}\right)^{\nu_{2}-(m+1) / 2} \\
& \quad \cdot{ }_{2} F_{1}\left(\beta_{2}-\alpha_{2}, \nu_{1}+v_{2} ; \beta_{2} ; I_{m}-R_{1}\right), \quad 0<R_{1}<I_{m}
\end{aligned}
$$

and the p.d.f. of $S_{1}=X_{1}+X_{2}$ is given by

$$
\begin{array}{r}
\frac{\theta^{-m\left(\nu_{1}+\nu_{2}\right)} \Gamma_{m}\left(\alpha_{2}\right) \Gamma_{m}\left(\beta_{2}-v_{2}\right)}{\Gamma_{m}\left(\beta_{2}\right) \Gamma_{m}\left(\alpha_{2}-v_{2}\right) \Gamma_{m}\left(\nu_{1}+v_{2}\right)} \operatorname{det}\left(S_{1}\right)^{\nu_{1}+v_{2}-(m+1) / 2} \\
\cdot \operatorname{etr}\left(-\frac{1}{\theta} S_{1}\right){ }_{2} F_{2}\left(\nu_{2}, \beta_{2}-\alpha_{2} ; v_{1}+\nu_{2}, \beta_{2} ; \frac{1}{\theta} S_{1}\right), \\
S_{1}>0 .
\end{array}
$$

Proof. Interchanging subscripts 1 and 2 in Theorem 22, the p.d.f. of $R_{2}=\left(X_{1}+X_{2}\right)^{-1 / 2} X_{2}\left(X_{1}+X_{2}\right)^{-1 / 2}$ is given by

$$
\begin{aligned}
& \frac{\Gamma_{m}\left(\alpha_{2}\right) \Gamma_{m}\left(\beta_{2}-v_{2}\right) \Gamma_{m}\left(\nu_{1}+v_{2}\right)}{\Gamma_{m}\left(\beta_{2}\right) \Gamma_{m}\left(\alpha_{2}-v_{2}\right) \Gamma_{m}\left(\nu_{1}\right) \Gamma_{m}\left(v_{2}\right)} \operatorname{det}\left(R_{2}\right)^{v_{2}-(m+1) / 2} \\
& \cdot \operatorname{det}\left(I_{m}-R_{2}\right)^{\nu_{2}-(m+1) / 2} \\
& \quad \cdot{ }_{2} F_{1}\left(\beta_{2}-\alpha_{2}, v_{1}+v_{2} ; \beta_{2} ; R_{2}\right), \quad 0<R_{2}<I_{m},
\end{aligned}
$$

where now $X_{1} \sim \mathrm{Ga}\left(m, v_{1}, \theta\right)$ and $X_{2} \sim \mathrm{CH}_{m}\left(\nu_{2}, \alpha_{2}, \beta_{2}, \theta\right.$, kind 1). The desired result is now obtained by observing that $R_{2}=I_{m}-R_{1}$. Similarly, the p.d.f. of $S_{1}$ is obtained by interchanging subscripts 1 and 2 in the p.d.f. of $S$.

Corollary 24. Let the random matrices $X_{1}$ and $X_{2}$ be independent, $X_{1} \sim \mathrm{CH}_{m}\left(\nu_{1}, \alpha_{1}, \nu_{1}+v_{2}, \theta\right.$, kind 1) and $X_{2} \sim \operatorname{Ga}\left(m, v_{2}, \theta\right)$. Then, $\left(X_{1}+X_{2}\right)^{-1 / 2} X_{1}\left(X_{1}+X_{2}\right)^{-1 / 2} \sim$ $\operatorname{B1}\left(m, v_{1}, \alpha_{1}-v_{1}\right)$.

Proof. The desired result is obtained by substituting $\beta_{1}=\nu_{1}+$ $v_{2}$ in the p.d.f. of $R$ and simplifying the resulting expression by using (16).

Corollary 25. Let the random matrices $X_{1}$ and $X_{2}$ be independent, $X_{1} \sim \mathrm{Ga}\left(m, v_{1}, \theta\right)$ and $X_{2} \sim \mathrm{CH}\left(m, v_{2}, \alpha_{2}, v_{1}+\right.$ $\nu_{2}, \theta$, kind 1). Then, $\left(X_{1}+X_{2}\right)^{-1 / 2} X_{1}\left(X_{1}+X_{2}\right)^{-1 / 2} \sim$ $\operatorname{B} 1\left(m, \alpha_{2}-v_{2}, v_{2}\right)$.

Proof. The desired result is obtained by substituting $\beta_{2}=\nu_{1}+$ $\nu_{2}$ in the p.d.f. of $R_{1}$ and simplifying the resulting expression by using (16).

Corollary 26. Let the random matrices $X_{1}$ and $X_{2}$ be independent, $X_{1} \sim \mathrm{CH}_{m}\left(\nu_{1}, \alpha_{1}, \alpha_{1}+\nu_{1}+\nu_{2}, \theta\right.$, kind 1) and $X_{2} \sim$ $\mathrm{Ga}\left(m, v_{2}, \theta\right)$. Then $X_{1}+X_{2} \sim \mathrm{CH}_{m}\left(\nu_{1}+v_{2}, \alpha_{1}+v_{2}, \alpha_{1}+v_{1}+\right.$ $\nu_{2}, \theta$, kind 1 ).

Proof. The result is obtained by substituting $\beta_{1}=\alpha_{1}+\nu_{1}+\nu_{2}$ in the p.d.f. of $S$ and simplifying the resulting expression by using (15). 
Corollary 27. Let the random matrices $X_{1}$ and $X_{2}$ be independent, $X_{1} \sim \mathrm{Ga}\left(m, \nu_{1}, \theta\right)$ and $X_{2} \sim \mathrm{CH}_{m}\left(\nu_{2}, \alpha_{2}, \alpha_{2}+\nu_{1}+\right.$ $v_{2}, \theta$, kind 1). Then $X_{1}+X_{2} \sim \mathrm{CH}_{m}\left(\nu_{1}+v_{2}, \alpha_{2}+v_{1}, \alpha_{2}+v_{1}+\right.$ $\nu_{2}, \theta$, kind 1 ).

Proof. The result is obtained by substituting $\beta_{2}=\alpha_{2}+\nu_{1}+v_{2}$ in the p.d.f. of $S$ and simplifying the resulting expression by using (15).

Corollary 28. Let the random matrices $X_{1}$ and $X_{2}$ be independent, $X_{1} \sim \mathrm{Ga}\left(m, v_{1}, \theta\right)$ and $X_{2} \sim \mathrm{Ga}\left(m, v_{2}, \theta\right)$. Then, $\left(X_{1}+X_{2}\right)^{-1 / 2} X_{1}\left(X_{1}+X_{2}\right)^{-1 / 2} \sim \mathrm{B} 1\left(m, v_{1}, v_{2}\right)$ and $X_{1}+X_{2} \sim$ $\mathrm{Ga}\left(m, v_{1}+v_{2}, \theta\right)$.

Proof. Substitute $\beta_{1}=\alpha_{1}$ in the p.d.f. of $R$ or $\beta_{2}=\alpha_{2}$ in the p.d.f. of $R_{1}$ and simplify the resulting expression to get the desired result.

\section{Related Distributions}

This section gives distributional results for the determinant of the random matrix distributed as confluent hypergeometric function kind 1 .

In an unpublished report, Coelho et al. [13] have shown that if $z$ is a positive random variable and $E\left(z^{h}\right)$ is defined for $h$ in some neighborhood of zero, then the moments $E\left(z^{h}\right)$ uniquely identify the distribution of $z$. In the next theorem, we will use this result to derive the distribution of the product of two independent confluent hypergeometric function kind 1 variables.

Theorem 29. If $x_{1} \sim \mathrm{CH}\left(\nu, \alpha, \beta, \theta\right.$, kind 1) and $x_{2} \sim \mathrm{CH}(\nu+$ $1 / 2, \alpha+1, \beta+1, \theta$, kind 1) are independent, then $2 \sqrt{x_{1} x_{2}} \sim$ $\mathrm{CH}(2 \nu, 2 \alpha, 2 \beta, \theta$, kind 1$)$.

Proof. The $h$ th moment of $2 \sqrt{x_{1} x_{2}}$ is derived as

$$
\begin{aligned}
E[ & \left.\left(2 \sqrt{x_{1} x_{2}}\right)^{h}\right] \\
& =\frac{(2 \theta)^{h} \Gamma(\beta-v) \Gamma(\nu+h / 2) \Gamma(\alpha-v-h / 2)}{\Gamma(\nu) \Gamma(\alpha-v) \Gamma(\beta-v-h / 2)} \\
& \cdot \frac{\Gamma(\beta-v+1 / 2) \Gamma(\nu+1 / 2+h / 2) \Gamma(\alpha-v+1 / 2-h / 2)}{\Gamma(\nu+1 / 2) \Gamma(\alpha-v+1 / 2) \Gamma(\beta-v+1 / 2-h / 2)} .
\end{aligned}
$$

Now, using the duplication formula for gamma function, namely,

$$
\Gamma(2 z)=\frac{\Gamma(z) \Gamma(z+1 / 2)}{2^{1-2 z} \sqrt{\pi}},
$$

the $h$ th moment of $2 \sqrt{x_{1} x_{2}}$ is rewritten as

$$
\begin{aligned}
& E\left[\left(2 \sqrt{x_{1} x_{2}}\right)^{h}\right] \\
& =\frac{\theta^{h} \Gamma(2 \beta-2 \nu) \Gamma(2 \nu+h) \Gamma(2 \alpha-2 \nu-h)}{\Gamma(2 \nu) \Gamma(2 \alpha-2 \nu) \Gamma(2 \beta-2 \nu-h)},
\end{aligned}
$$

where $v>0, \alpha-v>0, \beta-v>0,2 v>h, 2(\alpha-v)>h$, and $2(\beta-\nu)>h$.
Finally, comparison of the above expression with the one given in (45) yields the desired result.

Theorem 30. If $X \sim \mathrm{CH}_{m}(\nu, \alpha, \beta, \Omega, \theta$, kind 1), then $\operatorname{det}\left(\Omega^{-1 / 2} X \Omega^{-1 / 2}\right)$ is distributed as $\prod_{i=1}^{m} z_{i}$, where $z_{1}, \ldots, z_{m}$ are independent, $z_{i} \sim \mathrm{CH}(\nu-(i-1) / 2, \alpha-i+1, \beta-i+1, \theta$, kind 1$), i=1, \ldots, m$.

Proof. Writing multivariate gamma functions in terms of ordinary gamma function, (42) is rewritten as

$$
\begin{aligned}
& E\left[\operatorname{det}\left(\Omega^{-1 / 2} X \Omega^{-1 / 2}\right)^{h}\right] \\
& =\prod_{i=1}^{m}\left[\frac{\theta^{h} \Gamma[\beta-\nu-(i-1) / 2]}{\Gamma[\nu-(i-1) / 2] \Gamma[\alpha-\nu-(i-1) / 2]}\right. \\
& \left.\quad . \frac{\Gamma[\nu-(i-1) / 2+h] \Gamma[\alpha-\nu-(i-1) / 2-h]}{\Gamma[\beta-\nu-(i-1) / 2-h]}\right] .
\end{aligned}
$$

Now, comparing the above expression with (45), we get $E\left[\operatorname{det}\left(\Omega^{-1 / 2} X \Omega^{-1 / 2}\right)^{h}\right]=\prod_{i=1}^{m} E\left(z_{i}^{h}\right)$.

Corollary 31. If $X \sim \mathrm{CH}_{2}(\nu, \alpha, \beta, \theta$, kind 1), then

$$
2 \operatorname{det}(X)^{1 / 2} \sim \mathrm{CH}(2 \nu-1,2 \alpha-2,2 \beta-2, \theta \text {, kind } 1) .
$$

Proof. For $m=2, \operatorname{det}(X)$ is distributed as $z_{1} z_{2}$, where $z_{1}$ and $z_{2}$ are independent, $z_{1} \sim \mathrm{CH}(\nu, \alpha, \beta, \theta$, kind 1$)$ and $z_{2} \sim$ $\mathrm{CH}(\nu-1 / 2, \alpha-1, \beta-1, \theta$, kind 1$)$. From Theorem 29 , we have $2 \sqrt{z_{1} z_{2}} \sim \mathrm{CH}(2 \nu-1,2 \alpha-2,2 \beta-2, \theta$, kind 1$)$.

Corollary 32. If $X \sim \mathrm{Ga}(m, v, \theta, \Omega)$, then $\operatorname{det}\left(\Omega^{-1 / 2} X \Omega^{-1 / 2}\right)$ is distributed as $\prod_{i=1}^{m} z_{i}$, where $z_{1}, \ldots, z_{m}$ are independent, $z_{i} \sim$ $\mathrm{Ga}(\nu-(i-1) / 2, \theta), i=1, \ldots, m$.

\section{Distribution of Eigenvalues}

In this section, we derive density of eigenvalues of random matrix distributed as confluent hypergeometric function kind 1.

Theorem 33. Let $A$ be a positive definite random matrix of order $m$ with the p.d.f. $f(A)$. Then, the joint p.d.f. of the eigenvalues $l_{1}, l_{2}, \ldots, l_{m}$ of $A$ is given by

$$
\frac{\pi^{m^{2} / 2}}{\Gamma_{m}(m / 2)} \prod_{i<j}^{m}\left(l_{i}-l_{j}\right) \int_{O(m)} f\left(H L H^{\prime}\right)(d H),
$$

where $l_{1}>l_{2}>\cdots>l_{m}>0, L=\operatorname{diag}\left(l_{1}, l_{2}, \ldots, l_{m}\right)$, and $(d H)$ is the unit invariant Haar measure on the group of orthogonal matrices $O(m)$.

Proof of Theorem 33 and several other results can be found in Muirhead [7]. 
Theorem 34. If $X \sim \mathrm{CH}_{m}(\nu, \alpha, \beta, \theta, \Omega$, kind 1$)$, then the joint p.d.f. of the eigenvalues $x_{1}, x_{2}, \ldots, x_{m}$ of $X$ is given by

$$
\begin{gathered}
\frac{\pi^{m^{2} / 2} \Gamma_{m}(\alpha) \Gamma_{m}(\beta-\nu) \operatorname{det}(\theta \Omega)^{-v}}{\Gamma_{m}(m / 2) \Gamma_{m}(\nu) \Gamma_{m}(\beta) \Gamma_{m}(\alpha-\nu)}\left[\prod_{i<j}^{m}\left(x_{i}-x_{j}\right)\right] \\
\cdot \prod_{i=1}^{m}\left[x_{i}^{\nu-(m+1) / 2}\right]_{1} F_{1}^{(m)}\left(\alpha ; \beta ;-\frac{1}{\theta} \Omega^{-1}, L\right),
\end{gathered}
$$

where $0<x_{m}<\cdots<x_{1}<\infty, L=\operatorname{diag}\left(x_{1}, \ldots, x_{m}\right)$, and ${ }_{1} F_{1}^{(m)}$ is the two-matrix argument confluent hypergeometric function.

Proof. The p.d.f. of $X$ is given by (5). Applying Theorem 33, we obtain the joint p.d.f. of the eigenvalues $x_{1}, \ldots, x_{m}$ of $X$ as

$$
\begin{gathered}
\frac{\pi^{m^{2} / 2} \Gamma_{m}(\alpha) \Gamma_{m}(\beta-\nu) \operatorname{det}(\theta \Omega)^{-\nu}}{\Gamma_{m}(m / 2) \Gamma_{m}(\nu) \Gamma_{m}(\beta) \Gamma_{m}(\alpha-\nu)}\left[\prod_{i<j}^{m}\left(x_{i}-x_{j}\right)\right] \\
\cdot \prod_{i=1}^{m}\left[x_{i}^{\nu-(m+1) / 2}\right] \\
\cdot \int_{O(m)}{ }_{1} F_{1}^{(m)}\left(\alpha ; \beta ;-\frac{1}{\theta} \Omega^{-1} H L H^{\prime}\right)(\mathrm{d} H) .
\end{gathered}
$$

Now, using (28), we obtain the desired result.

\section{A Generalized Form}

In this section, we give a more general form of the matrix variate confluent hypergeometric function kind 1 distribution by introducing an additional factor $\operatorname{etr}\left(-\Psi^{-1} X / \theta\right)$ in the p.d.f. (5). The p.d.f. of $X$, in this case, is given by

$$
\begin{gathered}
\frac{\theta^{-m \nu} \operatorname{det}\left(\Psi^{-1}+\Omega^{-1}\right)^{\nu}}{\Gamma_{m}(\nu)_{2} F_{1}\left(\nu, \beta-\alpha ; \beta ;\left(\Psi^{-1}+\Omega^{-1}\right)^{-1} \Omega^{-1}\right)} \operatorname{etr}\left(-\frac{1}{\theta}\right) \\
\cdot \operatorname{det}(X)^{\nu-(m+1) / 2}{ }_{1} F_{1}\left(\alpha ; \beta ;-\frac{1}{\theta} \Omega^{-1} X\right),
\end{gathered}
$$

where $X>0$. We will write $X \sim \mathrm{CH}_{m}(\nu, \alpha, \beta, \theta, \Omega, \Psi$, kind 1) if the density of $X$ is given by (82). For $\Psi=I_{m}$ and $\theta=1$, the above p.d.f. reduces to

$$
\begin{aligned}
& \frac{\operatorname{det}\left(I_{m}+\Omega^{-1}\right)^{\nu}}{\Gamma_{m}(\nu){ }_{2} F_{1}\left(\nu, \beta-\alpha ; \beta ;\left(I_{m}+\Omega\right)^{-1}\right)} \operatorname{etr}(-X) \\
& \cdot \operatorname{det}(X)^{\nu-(m+1) / 2}{ }_{1} F_{1}\left(\alpha ; \beta ;-\Omega^{-1} X\right), \quad X>0,
\end{aligned}
$$

which is a special case of the generalized hypergeometric function density defined by Roux [14].

Theorem 35. Let $Z \mid \Sigma \sim \operatorname{InvGa}\left(m, \mu, \theta, \Sigma^{-1}\right)$. Further, let the prior distribution of $\Sigma$ be a generalized matrix variate confluent hypergeometric kind 1 distribution with parameters $\nu, \alpha, \beta, \theta, \Omega$, and $\Psi, \Sigma \sim \mathrm{CH}_{m}(\nu, \alpha, \beta, \theta, \Omega, \Psi$, kind 1). Then, the marginal distribution of $Z$ is a generalized inverted matrix variate beta with the density

$$
\begin{aligned}
& m(Z) \\
& =\frac{\Gamma_{m}(\mu+\nu) \operatorname{det}\left(\Psi^{-1}+\Omega^{-1}\right)^{\nu}}{\Gamma_{m}(\nu) \Gamma_{m}(\mu)_{2} F_{1}\left(\nu, \beta-\alpha ; \beta ;\left(\Psi^{-1}+\Omega^{-1}\right)^{-1} \Omega^{-1}\right)} \\
& \quad \cdot \frac{\operatorname{det}(Z)^{-\mu-(m+1) / 2}}{\operatorname{det}\left(\Psi^{-1}+\Omega^{-1}+Z^{-1}\right)^{\mu+\nu}} \\
& \quad \cdot{ }_{2} F_{1}\left(\mu+\nu, \beta-\alpha ; \beta ;\left(\Psi^{-1}+\Omega^{-1}+Z^{-1}\right)^{-1} \Omega^{-1}\right),
\end{aligned}
$$

where $Z>0$.

Proof. By definition, the marginal density of $Z$, denoted by $m(Z)$, is obtained as

$$
m(Z)=\int_{\Sigma>0} f(Z \mid \Sigma) \pi(\Sigma) \mathrm{d} \Sigma .
$$

Now, substituting for $f(Z \mid \Sigma)$ and $\pi(\Sigma)$, we get

$$
\begin{aligned}
& m(Z) \\
& =\frac{\theta^{-m(\nu+\mu)} \operatorname{det}\left(\Psi^{-1}+\Omega^{-1}\right)^{\nu}}{\Gamma_{m}(\nu) \Gamma_{m}(\mu)_{2} F_{1}\left(\nu, \beta-\alpha ; \beta ;\left(\Psi^{-1}+\Omega^{-1}\right)^{-1} \Omega^{-1}\right)} \\
& \cdot \operatorname{det}(Z)^{-\mu-(m+1) / 2} \int_{\Sigma>0} \operatorname{det}(\Sigma)^{\mu+\nu-(m+1) / 2} \\
& \cdot \operatorname{etr}\left[-\frac{1}{\theta}\left(\Psi^{-1}+\Omega^{-1}+Z^{-1}\right) \Sigma\right] \\
& \quad \cdot{ }_{1} F_{1}\left(\beta-\alpha ; \beta ; \frac{1}{\theta} \Omega^{-1} \Sigma\right) \mathrm{d} \Sigma .
\end{aligned}
$$

Finally, evaluating the above expression by using (21) and simplifying, we get the desired result.

Theorem 36. Let $Z \mid \Sigma \sim \operatorname{InvGa}\left(m, \mu, \Sigma^{-1}\right)$. Further, let the prior distribution of $\Sigma$ be a generalized matrix variate confluent hypergeometric function kind 1 distribution parameters $\nu, \alpha$, $\beta, \theta, \Omega$, and $\Psi, \Sigma \sim \mathrm{CH}_{m}(\nu, \alpha, \beta, \theta, \Omega, \Psi$, kind 1). Then, the posterior distribution of $\Sigma$ is a generalized matrix variate confluent hypergeometric kind 1 distribution with parameters $\mu+\nu, \alpha, \beta, \theta, \Omega$, and $\left(\Psi^{-1}+Z^{-1}\right)^{-1}$.

Proof. By definition and Theorem 35, we have

$$
\pi(\Sigma \mid Z)=\frac{f(Z \mid \Sigma) \pi(\Sigma)}{m(Z)} .
$$

Now, substituting appropriately, we get

$$
\begin{aligned}
& \pi(\Sigma \mid Z) \\
& =\frac{\theta^{-m(\nu+\mu)} \operatorname{det}\left(\Psi^{-1}+\Omega^{-1}+Z^{-1}\right)^{\nu+\mu}}{\Gamma_{m}(\nu+\mu){ }_{2} F_{1}\left(\nu+\mu, \beta-\alpha ; \beta ;\left(\Psi^{-1}+\Omega^{-1}+Z^{-1}\right)^{-1} \Omega^{-1}\right)} \\
& \quad \cdot \operatorname{etr}\left[-\frac{1}{\theta}\left(\Psi^{-1}+Z^{-1}\right) \Sigma\right] \operatorname{det}(\Sigma)^{\nu+\mu-(m+1) / 2} \\
& \quad \cdot{ }_{1} F_{1}\left(\alpha ; \beta ;-\frac{1}{\theta} \Omega^{-1} \Sigma\right), \quad \Sigma>0,
\end{aligned}
$$

which is the desired result. 
From the above results it is quite clear that the generalized matrix variate confluent hypergeometric function kind 1 distribution as the prior distribution is conjugate. Thus, this distribution may be used as an alternative to matrix variate gamma distribution.

\section{Conflict of Interests}

The authors declare that there is no conflict of interests regarding the publication of this paper.

\section{Acknowledgment}

The research work of Daya K. Nagar was supported by the Sistema Universitario de Investigación, Universidad de Antioquia, by Project no. IN10164CE.

\section{References}

[1] A. K. Gupta and D. K. Nagar, Matrix Variate Distributions, Chapman \& Hall/CRC Press, Boca Raton, Fla, USA, 2000.

[2] G. J. van der Merwe and J. J. J. Roux, "On a generalized matrixvariate hypergeometric distribution," South African Statistical Journal, vol. 8, pp. 49-58, 1974.

[3] J. M. Orozco-Castañeda, D. K. Nagar, and A. K. Gupta, "Generalized bivariate beta distributions involving Appell's hypergeometric function of the second kind," Computers and Mathematics with Applications, vol. 64, no. 8, pp. 2507-2519, 2012.

[4] Y. L. Luke, The Special Functions and Their Approximations, vol. 1, Academic Press, New York, NY, USA, 1969.

[5] A. G. Constantine, "Some non-central distribution problems in multivariate analysis," Annals of Mathematical Statistics, vol. 34, pp. 1270-1285, 1963.

[6] A. T. James, "Distributions of matrix variates and latent roots derived from normal samples," Annals of Mathematical Statistics, vol. 35, pp. 475-501, 1964.

[7] R. J. Muirhead, Aspects of Multivariate Statistical Theory, Wiley Series in Probability and Mathematical Statistics, John Wiley \& Sons, New York, NY, USA, 1982.

[8] H. Hashiguchi, Y. Numata, N. Takayama, and A. Takemura, "The holonomic gradient method for the distribution function of the largest root of a Wishart matrix," Journal of Multivariate Analysis, vol. 117, pp. 296-312, 2013.

[9] P. Koev and A. Edelman, "The efficient evaluation of the hypergeometric function of a matrix argument," Mathematics of Computation, vol. 75, no. 254, pp. 833-846, 2006.

[10] A. Iranmanesh, M. Arashi, D. K. Nagar, and S. M. Tabatabaey, "On inverted matrix variate gamma distribution," Communications in Statistics-Theory and Methods, vol. 42, no. 1, pp. 28-41, 2013.

[11] J. J. Roux and G. J. van der Merwe, "Families of multivariate distributions having properties usually associated with the Wishart distribution," South African Statistical Journal, vol. 8, pp. 111-117, 1974.

[12] C. G. Khatrzi, "On certain distribution problems based on positive definite quadratic functions in normal vectors," Annals of Mathematical Statistics, vol. 37, pp. 468-479, 1966.

[13] C. A. Coelho, R. P. Alberto, and L. M. Grilo, "When do the moments uniquely identify a distribution," CMA 13-2005, Centro de Matemática e Aplicações, Departamento de Matemática,
Faculdade de Ciências e Tecnologia, Universidade Nova de Lisboa, 2005.

[14] J. J. J. Roux, "On generalized multivariate distributions. South African Statist," South African Statistical Journal, vol. 5, pp. 91100, 1971. 


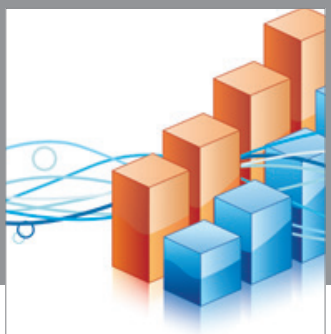

Advances in

Operations Research

vatem alat4

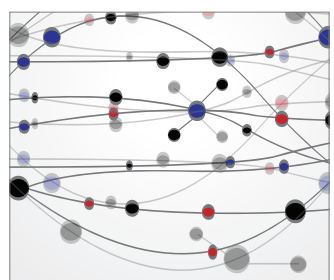

\section{The Scientific} World Journal
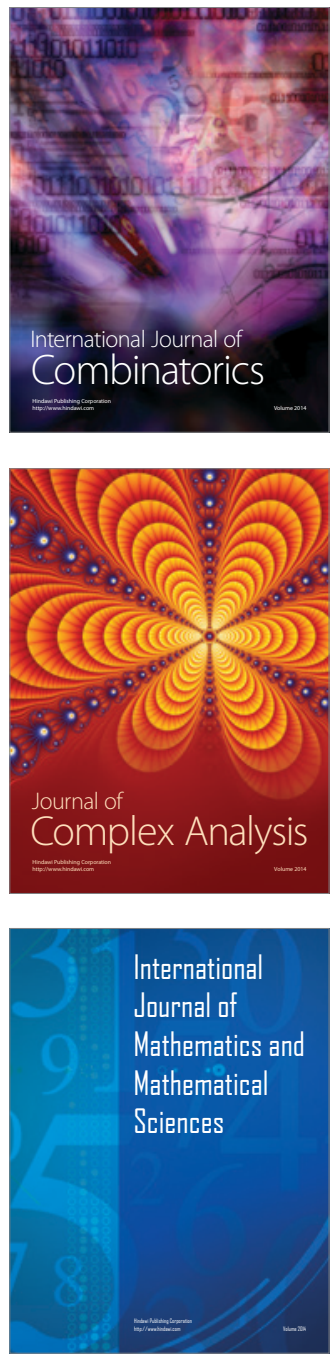
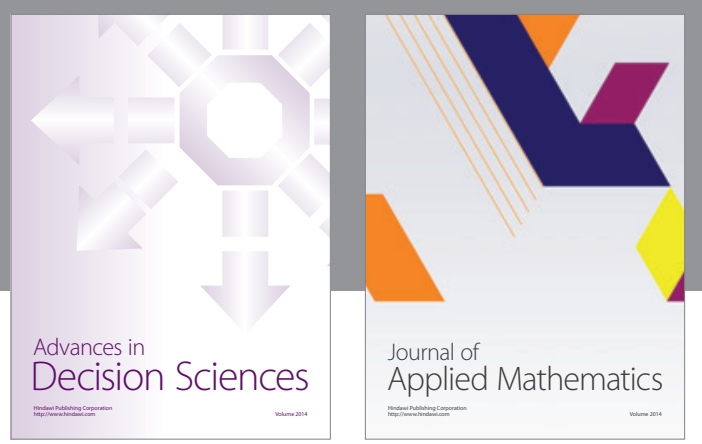

Algebra

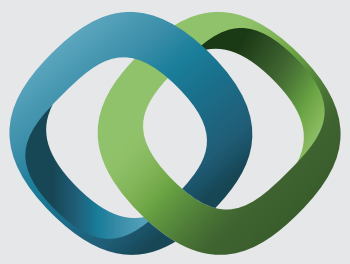

\section{Hindawi}

Submit your manuscripts at

http://www.hindawi.com
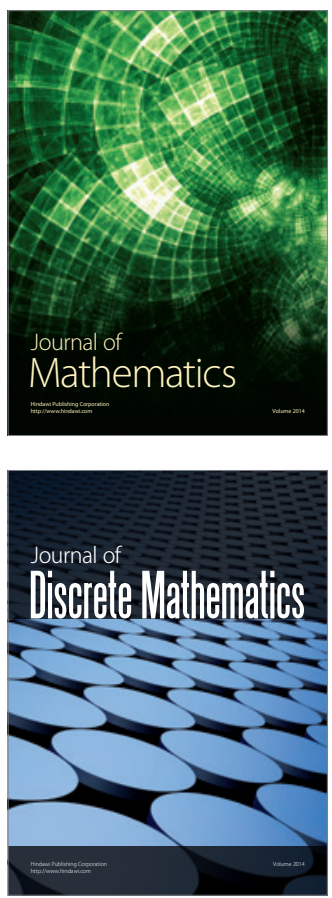

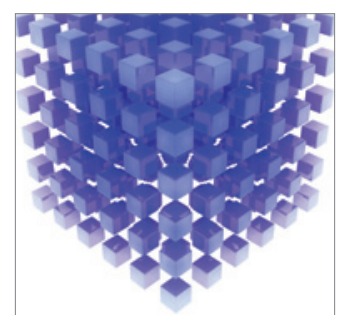

Mathematical Problems in Engineering
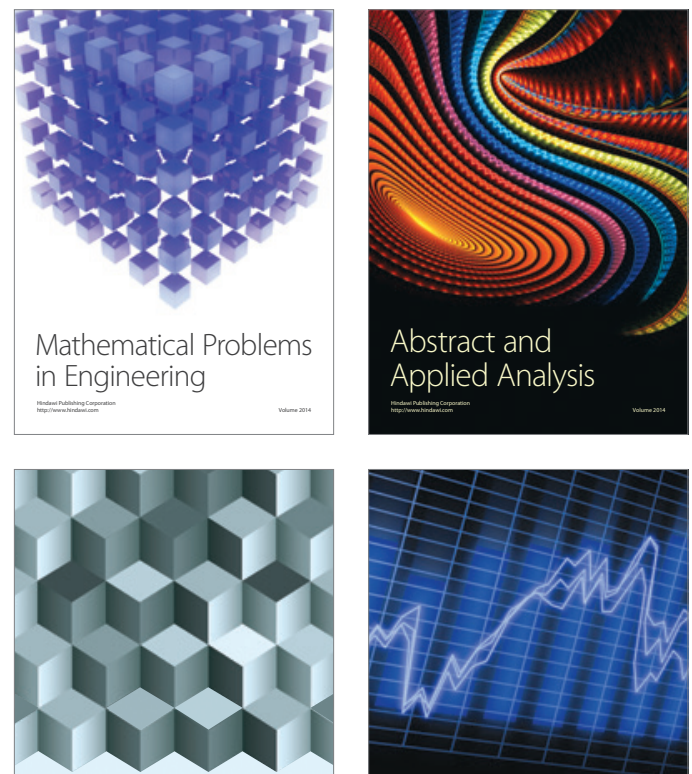

Journal of

Function Spaces

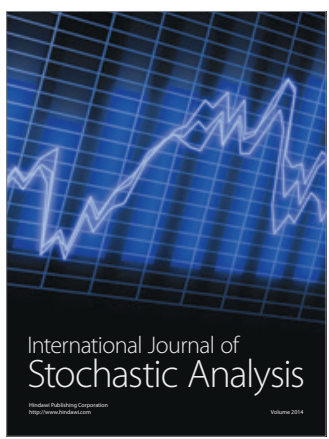

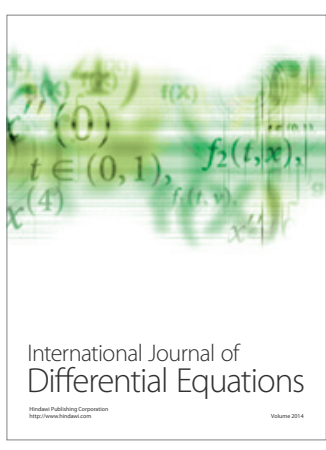
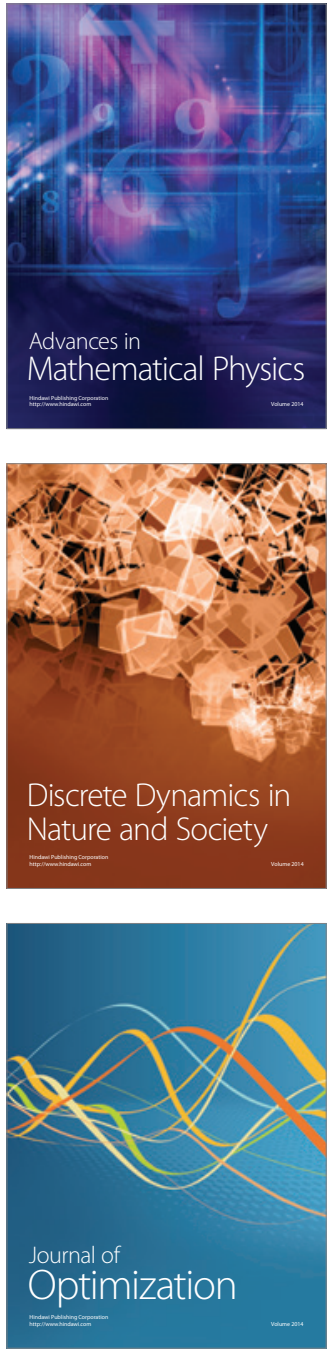